\title{
HÖLDER CONTINUITY FOR NONLINEAR ELLIPTIC PROBLEM IN MUSIELAK-ORLICZ-SOBOLEV SPACE
}

\author{
BEIBEI WANG, DUCHAO LIU, AND PEIHAO ZHAO
}

\begin{abstract}
Under appropriate assumptions on the $N(\Omega)$-fucntion, the De Giorgi process is presented in the framework of Musielak-Orlicz-Sobolev space to prove the Hölder continuity of fully nonlinear elliptic problems. As the applications, the Hölder continuity of the minimizers for a class of the energy functionals in Musielak-Orlicz-Sobolev spaces is proved; and furthermore, the Hölder continuity of the weak solutions for a class of fully nonlinear elliptic equations is provided.
\end{abstract}

\section{INTRODUCTION}

Since Ladyzhenskaya and Ural'tseva developed the method pioneered by De Giorgi [10] and introduced the class $\mathscr{B}\left(\Omega, M, \gamma, \gamma_{1}, \delta, 1 / q\right)$ (see [23]), through which the Hölder continuity of functions of this class can be proved, the class $\mathscr{B}(\cdots)$ in the case of the standard $m$-growth conditions was also proved working (e.g. $19,21,23,35)$. This method is also available to investigate the variational problems and the regularity of solutions of quasi-linear elliptic equations. The regularity of solutions under nonstandard growth conditions is investigated following an counterexample given by Giaquinta in 1987 [20, further relevant contributions are for example in [2, 3, 31, 32, 33, 24, 25, 26, 15, 27, 37, 38, and a more recent paper 22.

Our aim in the current paper is to study the Hölder continuity of the minimizers for functionals defined on the Musielak-Orlicz-Sobolev spaces and the Hölder continuity of the weak solutions for the associated fully nonlinear elliptic equations, mainly in the framework of Musielak-Orlicz-Sobolev space and from the viewpoint of PDEs.

In fact, there are some important classical regularity results for the minimizers of integral functionals within the Sobolev, variable exponent, Orlicz and MusielakOrlicz-Sobolev framework settings in the literature.

In an early work of [1], Acerbi and Fusco proved that for any $W_{\text {loc }}^{1, p}\left(\Omega ; \mathbb{R}^{N}\right)$ local minimizer $u$ with $1<p<2$ of the integral functional $\int f(x, v(x), D v(x)) \mathrm{d} x$, its gradient $D u$ is actually locally $\lambda$-Hölder continuous for some $\lambda>0$ when $f$ fulfills some uniformly $p$-exponent increasing conditions. In the recent papers [8, 7, Colombo and Mingione investigated the regularity of $W^{1, p}\left(\Omega ; \mathbb{R}^{N}\right)$-local minimizer $u$ of the integral functional defined by $\mathcal{P}_{p, q}(w, \Omega)=\int_{\Omega}\left(|D w|^{p}+a(x)|D w|^{q}\right) \mathrm{d} x$ where $0 \leq a(x) \leq L$ and $1<p<q$. In fact, the authors proved that $D u$ is actually locally Hölder continuous when $0 \leq a(\cdot) \in C^{0, \alpha}(\Omega)$ and $\frac{q}{p}<1+\frac{\alpha}{n}$. The results in [8]

\footnotetext{
*In memory of Professor Wenyuan Chen.

${ }^{\dagger}$ Research supported by the National Natural Science Foundation of China (NSFC 11501268 and NSFC 11471147).

1991 Mathematics Subject Classification. 35B38, 35D05, 35J20.

Key words and phrases. Musielak-Orlicz-Sobolev space; Local Bounded property.
} 
cover more general functionals than $\mathcal{P}_{p, q}$ in [7. As described in [8], the result was actually extended to the vector case and a larger class of more general functionals. When considering the particular case $p=q$ or $a(x) \equiv 0$, the regularity theory of minimizers is by now well understood, see for instance [30, 29, 34. In a much more recent paper [6], the authors considered the minimizer $u \in W^{1,1}(\Omega)$ of the functional defined by $\mathcal{P}_{\log }(w, \Omega):=\int_{\Omega}\left[|D w|^{p}+a(x)|D w|^{p} \log (e+|D w|)\right] \mathrm{d} x$, in which the function $a(\cdot)$ is nonnegative, bounded and satisfies $|a(x)-a(y)| \leq w(|x-y|)$ for every $x, y \in \Omega$. They proved that if $l:=\limsup _{r \rightarrow 0} w(r) \log \left(\frac{1}{r}\right)<\infty$, then $u \in C_{l o c}^{1, \beta}$ for some $\beta \in(0,1)$; if $l=0$, then $u \in C_{l o c}^{1, \beta}$ for every $\beta \in(0,1)$; if $w(r) \lesssim r^{\sigma}$ with $\sigma \in(0,1)$ then $D u$ is locally Hölder continuous in $\Omega$.

We point out that some regularity results in the variable exponent spaces framework can be found in the works [9, 16, 11. In the paper 9], Coscia and Mingione proved that for any $W^{1,1}(\Omega)$-local minimizer $u$ of the integral functional $\int_{\Omega}|\nabla u|^{p(x)} \mathrm{d} x$, its gradient $D u$ is actually locally Hölder continuous when $p(\cdot)$ is locally Hölder continuous in $\Omega$. When $p(\cdot)$ satisfies $R^{-\operatorname{osc}\left\{p ; B_{R}\right\}} \leq L$, for all $B_{R} \subset \Omega$, Fan and Zhao $\left[16\right.$ proved that $W^{1, p(\cdot)}$-minimizer $u \in C^{0, \beta}(\Omega)$ for some $\beta \in(0,1)$. In 11, Diening and Hästö also introduced in the important study [11] the TriebelLizorkin spaces with variable smoothness and itegrability, including a trace theorem in the variable index case. In [4, Adamowicz and collaborators showed the continuity of quasiminimizers of energy functionals $\int f(x, u, \nabla u) \mathrm{d} x$ when $f$ satisfies some uniformly $p(\cdot)$-exponent monotonicity assumptions.

In the recent works of [12, 13, Diening and his collaborators proved a series of regularity results in Orlicz spaces. More precisely, they proved in [12] the $C^{1, \alpha_{-}}$ regularity for local minimizers of functionals with $\varphi$-growth including the decay estimate, where $\varphi$ is a convex $C^{1}$-function independent of the parameter $x \in \Omega \subset$ $\mathbb{R}^{N}$; in [13, they established a local Lipschitz result for the local minimizers of asymptotically convex variational integrals.

For regularity results in the Musielak-Orlicz-Sobolev setting, we also noticed that Hästö and collaborators proved, in an important recent work [22, that Harnack's inequality still holds for quasi-minimizers in the Musielak-Orlicz-Sobolev spaces without any polynomial growth or coercivity conditions, which yields the local Hölder continuity of quasi-minimizers. Comparing with our current study, it is interesting to notice that we proposed a different monotonicity assumption for the $\Phi$ function from that in [22]'s. In our current study, we have proposed a more general uniformly monotonicity condition on the $N(\Omega)$ function. Meanwhile, with the regularity results in the key Theorem 3.1 we can prove not only the Hölder continuity of the minimizers for a more general class of energy functionals (see Section (4), but also the Hölder continuity of a kind of weak solutions for a class of fully nonlinear elliptic equations (see Section 5).

The paper is organized as follows. In Section 2, for the readers' convenience we recall some definitions and properties about Musielak-Orlicz-Sobolev spaces. In Section 3, we give some crucial lemmas in order to prove the main theorems of this paper. In Section 4 we prove the Hölder continuity of the minimizers of a class of the energy functionals in Musielak-Orlicz-Sobolev spaces. In Section 5, the Hölder continuity of the weak solution to a class of fully nonlinear elliptic equations is provided. 


\section{The Musielak-Orlicz-Sobolev Spaces}

In this section, we list some definitions and propositions related to MusielakOrlicz-Sobolev spaces. Firstly, we give the definition of $N$-function and generalized $N$-function as following.

Definition 2.1. A function $A: \mathbb{R} \rightarrow[0,+\infty)$ is called an $N$-function, denoted by $A \in N$, if $A$ is even and convex, $A(0)=0,0<A(t) \in C^{0}$ for $t \neq 0$, and the following conditions hold

$$
\lim _{t \rightarrow 0+} \frac{A(t)}{t}=0 \text { and } \lim _{t \rightarrow+\infty} \frac{A(t)}{t}=+\infty .
$$

Let $\Omega$ be a smooth domain in $\mathbb{R}^{n}$. A function $A: \Omega \times \mathbb{R} \rightarrow[0,+\infty)$ is called a generalized $N$-function, denoted by $A \in N(\Omega)$, if for each $t \in[0,+\infty)$, the function $A(\cdot, t)$ is measurable, and for a.e. $x \in \Omega$, we have $A(x, \cdot) \in N$.

Let $A \in N(\Omega)$, the Musielak-Orlicz space $L^{A}(\Omega)$ is defined by

$$
\begin{aligned}
L^{A}(\Omega):=\{u: u \text { is a measurable real function, and } \exists \lambda>0 \\
\text { such that } \left.\int_{\Omega} A\left(x, \frac{|u(x)|}{\lambda}\right) \mathrm{d} x<+\infty\right\}
\end{aligned}
$$

with the (Luxemburg) norm

$$
\|u\|_{L^{A}(\Omega)}=\|u\|_{A}:=\inf \left\{\lambda>0: \int_{\Omega} A\left(x, \frac{|u(x)|}{\lambda}\right) \mathrm{d} x \leq 1\right\} .
$$

The Musielak-Orlicz-Sobolev space $W^{1, A}(\Omega)$ can be defined by

$$
W^{1, A}(\Omega):=\left\{u \in L^{A}(\Omega):|\nabla u| \in L^{A}(\Omega)\right\}
$$

with the norm

where $\|\nabla u\|_{A}:=\||\nabla u|\|_{A}$.

$$
\|u\|_{W^{1, A}(\Omega)}=\|u\|_{1, A}:=\|u\|_{A}+\|\nabla u\|_{A},
$$

$A$ is called locally integrable if $A\left(\cdot, t_{0}\right) \in L_{\text {loc }}^{1}(\Omega)$ for every $t_{0}>0$.

Definition 2.2. We say that $a(x, t)$ is the Musielak derivative of $A(x, t) \in N(\Omega)$ at $t$ if for $x \in \Omega$ and $t \geq 0, a(x, t)$ is the right-hand derivative of $A(x, \cdot)$ at $t$; and for $x \in \Omega$ and $t \leq 0, a(x, t):=-a(x,-t)$.

Define $\widetilde{A}: \Omega \times \mathbb{R} \rightarrow[0,+\infty)$ by

$$
\widetilde{A}(x, s)=\sup _{t \in \mathbb{R}}(s t-A(x, t)) \text { for } x \in \Omega \text { and } s \in \mathbb{R} .
$$

$\widetilde{A}$ is called the complementary function to $A$ in the sense of Young. It is well known that if $A \in N(\Omega)$, then $\widetilde{A} \in N(\Omega)$ and $A$ is also the complementary function to $\widetilde{A}$.

For $x \in \Omega$ and $s \geq 0$, we denote by $a_{+}^{-1}(x, s)$ the right-hand derivative of $\widetilde{A}(x, \cdot)$ at $s$ at the same time define $a_{+}^{-1}(x, s)=-a_{+}^{-1}(x,-s)$ for $x \in \Omega$ and $s \leq 0$. Then for $x \in \Omega$ and $s \geq 0$, we have

$$
a_{+}^{-1}(x, s)=\sup \{t \geq 0: a(x, t) \leq s\}=\inf \{t>0: a(x, t)>s\} .
$$


Proposition 2.1. (See [17, 36]) Let $A \in N(\Omega)$. Then the following assertions hold.

(1) $A(x, t) \leq a(x, t) t \leq A(x, 2 t)$ for $x \in \Omega$ and $t \in \mathbb{R}$;

(2) $A$ and $\widetilde{A}$ satisfy the Young inequality

$$
s t \leq A(x, t)+\widetilde{A}(x, s) \text { for } x \in \Omega \text { and } s, t \in \mathbb{R}
$$

and the equality holds if $s=a(x, t)$ or $t=a_{+}^{-1}(x, s)$.

Let $A, B \in N(\Omega)$. We say that $A$ is weaker than $B$, denoted by $A \preccurlyeq B$, if there exist positive constants $K_{1}, K_{2}$ and $h \in L^{1}(\Omega) \cap L^{\infty}(\Omega)$ such that

$$
A(x, t) \leq K_{1} B\left(x, K_{2} t\right)+h(x) \text { for } x \in \Omega \text { and } t \in[0,+\infty) .
$$

Proposition 2.2. (See [17, 36]) Let $A, B \in N(\Omega)$ and $A \preccurlyeq B$. Then $\widetilde{B} \preccurlyeq \widetilde{A}$, $L^{B}(\Omega) \hookrightarrow L^{A}(\Omega)$ and $L^{\widetilde{A}}(\Omega) \hookrightarrow L^{\widetilde{B}}(\Omega)$.

Definition 2.3. We say that a function $A:[0,+\infty) \rightarrow[0,+\infty)$ satisfies the $\Delta_{2}(\Omega)$ condition, denoted by $A \in \Delta_{2}(\Omega)$, if there exist a positive constant $K>0$ and a nonnegative function $h \in L^{1}(\Omega)$ such that

$$
A(x, 2 t) \leq K A(x, t)+h(x) \text { for } x \in \Omega \text { and } t \in[0,+\infty) .
$$

If $A(x, t)=A(t)$ is an $N$-function and $h(x) \equiv 0$ in $\Omega$ in Definition 2.3 then $A \in \Delta_{2}(\Omega)$ if and only if $A$ satisfies the well-known $\Delta_{2}$ condition defined in [5, 14.

Proposition 2.3. (See [17]) Let $A \in N(\Omega)$ satisfy $\Delta_{2}(\Omega)$. Then the following assertions hold,

(1) $L^{A}(\Omega)=\left\{u: u\right.$ is a measurable function, and $\left.\int_{\Omega} A(x,|u(x)|) \mathrm{d} x<+\infty\right\}$;

(2) $\int_{\Omega} A(x,|u|) \mathrm{d} x<1$ (resp. $\left.=1 ;>1\right) \Longleftrightarrow\|u\|_{A}<1$ (resp. = $1 ;>1$ ), where $u \in L^{A}(\Omega)$;

(3) $\int_{\Omega} A\left(x,\left|u_{n}\right|\right) \mathrm{d} x \rightarrow 0$ (resp. $\left.1 ;+\infty\right) \Longleftrightarrow\left\|u_{n}\right\|_{A} \rightarrow 0$ (resp. $\left.1 ;+\infty\right)$, where $\left\{u_{n}\right\} \subset L^{A}(\Omega)$

(4) $u_{n} \rightarrow u$ in $L^{A}(\Omega) \Longrightarrow \int_{\Omega}\left|A\left(x,\left|u_{n}\right|\right) \mathrm{d} x-A(x,|u|)\right| \mathrm{d} x \rightarrow 0$ as $n \rightarrow \infty$;

(5) If $A^{\prime}$ also satisfies $\left(\Delta_{2}\right)$, then

$$
\left|\int_{\Omega} u(x) v(x) \mathrm{d} x\right| \leq 2\|u\|_{A}\|v\|_{\widetilde{A}}, \quad \forall u \in L^{A}(\Omega), v \in L^{\widetilde{A}}(\Omega) ;
$$

(6) $a(\cdot,|u(\cdot)|) \in L^{\widetilde{A}}(\Omega)$ for every $u \in L^{A}(\Omega)$.

The following assumptions will be used.

$\left(C_{1}\right) \inf _{x \in \Omega} A(x, 1)=c_{1}>0$;

Proposition 2.4. (See [17]) If $A \in N(\Omega)$ satisfies $\left(C_{1}\right)$, then $L^{A}(\Omega) \hookrightarrow L^{1}(\Omega)$ and $W^{1, A}(\Omega) \hookrightarrow W^{1,1}(\Omega)$. 
HÖLDER CONTINUITY FOR NONLINEAR ELLIPTIC PROBLEM IN MUSIELAK-ORLICZ-SOBOLEV SPACE *\$

Let $A \in N(\Omega)$ be locally integrable. We will denote

$$
\begin{aligned}
& W_{0}^{1, A}(\Omega):={\overline{C_{0}^{\infty}(\Omega)}}^{\|\cdot\|_{W^{1, A}(\Omega)}} \\
& \mathcal{D}_{0}^{1, A}(\Omega):={\overline{C_{0}^{\infty}(\Omega)}}^{\|\nabla \cdot\|_{L}{ }^{A}(\Omega)} .
\end{aligned}
$$

In the case that $\|\nabla u\|_{A}$ is an equivalent norm in $W_{0}^{1, A}(\Omega), W_{0}^{1, A}(\Omega)=\mathcal{D}_{0}^{1, A}(\Omega)$.

Proposition 2.5. (See [17]) Let $A \in N(\Omega)$ be locally integrable and satisfy $\left(C_{1}\right)$. Then

(1) the spaces $W^{1, A}(\Omega), W_{0}^{1, A}(\Omega)$ and $\mathcal{D}_{0}^{1, A}(\Omega)$ are separable Banach spaces, and

$$
\begin{gathered}
W_{0}^{1, A}(\Omega) \hookrightarrow W^{1, A}(\Omega) \hookrightarrow W^{1,1}(\Omega) \\
\mathcal{D}_{0}^{1, A}(\Omega) \hookrightarrow \mathcal{D}_{0}^{1,1}(\Omega)=W_{0}^{1,1}(\Omega) ;
\end{gathered}
$$

(2) the spaces $W^{1, A}(\Omega), W_{0}^{1, A}(\Omega)$ and $\mathcal{D}_{0}^{1, A}(\Omega)$ are reflexive provided $L^{A}(\Omega)$ is reflexive.

Proposition 2.6. (See [17) Let $A, B \in N(\Omega)$ and $A$ be locally integrable. If there is a compact imbedding $W^{1, A}(\Omega) \hookrightarrow \hookrightarrow L^{B}(\Omega)$ and $A \preccurlyeq B$, then there holds the following Poincaré inequality

$$
\|u\|_{A} \leq c\|\nabla u\|_{A}, \forall u \in W_{0}^{1, A}(\Omega)
$$

which implies that $\|\nabla \cdot\|_{A}$ is an equivalent norm in $W_{0}^{1, A}(\Omega)$ and $W_{0}^{1, A}(\Omega)=$ $\mathcal{D}_{0}^{1, A}(\Omega)$.

The following assumptions will be used.

$\left(P_{1}\right) \Omega \subset \mathbb{R}^{n}(n \geq 2)$ is a bounded domain with the cone property, and $A \in N(\Omega)$;

$\left(P_{2}\right) A: \bar{\Omega} \times \mathbb{R} \rightarrow[0,+\infty)$ is continuous and $A(x, t) \in(0,+\infty)$ for $x \in \bar{\Omega}$ and $t \in(0,+\infty)$.

Let $A$ satisfy $\left(P_{1}\right)$ and $\left(P_{2}\right)$. Denote by $A^{-1}(x, \cdot)$ the inverse function of $A(x, \cdot)$. We always assume that the following condition holds.

$\left(P_{3}\right) \quad A \in N(\Omega)$ and

$$
\int_{0}^{1} \frac{A^{-1}(x, t)}{t^{\frac{n+1}{n}}} \mathrm{~d} t<+\infty, \forall x \in \bar{\Omega}
$$

Under assumptions $\left(P_{1}\right),\left(P_{2}\right)$ and $\left(P_{3}\right)$, for each $x \in \bar{\Omega}$, the function $A(x, \cdot)$ : $[0,+\infty) \rightarrow[0,+\infty)$ is a strictly increasing homeomorphism. Define a function $A_{*}^{-1}: \bar{\Omega} \times[0,+\infty) \rightarrow[0,+\infty)$ by

$$
A_{*}^{-1}(x, s)=\int_{0}^{s} \frac{A^{-1}(x, \tau)}{\tau^{\frac{n+1}{n}}} \mathrm{~d} \tau \text { for } x \in \bar{\Omega} \text { and } s \in[0,+\infty) .
$$

Then under the assumption $\left(P_{3}\right), A_{*}^{-1}$ is well defined, and for each $x \in \bar{\Omega}, A_{*}^{-1}(x, \cdot)$ is strictly increasing, $A_{*}^{-1}(x, \cdot) \in C^{1}((0,+\infty))$ and the function $A_{*}^{-1}(x, \cdot)$ is concave.

Set

$$
T(x)=\lim _{s \rightarrow+\infty} A_{*}^{-1}(x, s), \forall x \in \bar{\Omega}
$$


Then $0<T(x) \leq+\infty$. Define an even function $A_{*}: \bar{\Omega} \times \mathbb{R} \rightarrow[0,+\infty)$ by

$$
A_{*}(x, t)= \begin{cases}s, & \text { if } x \in \bar{\Omega},|t| \in[0, T(x)) \text { and } A_{*}^{-1}(x, s)=|t|, \\ +\infty, & \text { for } x \in \bar{\Omega} \text { and }|t| \geq T(x) .\end{cases}
$$

Then if $A \in N(\Omega)$ and $T(x)=+\infty$ for any $x \in \bar{\Omega}$, it is well known that $A_{*} \in N(\Omega)$ (see [5]). $A_{*}$ is called the Sobolev conjugate function of $A$ (see $[5$ for the case of Orlicz functions).

Let $X$ be a metric space and $f: X \rightarrow(-\infty,+\infty]$ be an extended real-valued function. For $x \in X$ with $f(x) \in \mathbb{R}$, the continuity of $f$ at $x$ is well defined. For $x \in X$ with $f(x)=+\infty$, we say that $f$ is continuous at $x$ if given any $M>0$, there exists a neighborhood $U$ of $x$ such that $f(y)>M$ for all $y \in U$. We say that $f: X \rightarrow(-\infty,+\infty]$ is continuous on $X$ if $f$ is continuous at every $x \in X$. Define $\operatorname{Dom}(f)=\{x \in X: f(x) \in \mathbb{R}\}$ and denote by $C^{1-0}(X)$ the set of all locally Lipschitz continuous real-valued functions defined on $X$.

Remark 2.1. Suppose that $A \in N(\Omega)$ satisfy $\left(P_{2}\right)$, then for each $t_{0} \geq 0, \widetilde{A}\left(x, t_{0}\right)$, $A_{*}\left(x, t_{0}\right)$ are bounded.

The following assumptions will also be used.

$\left(P_{4}\right) T: \bar{\Omega} \rightarrow[0,+\infty]$ is continuous on $\bar{\Omega}$ and $T \in C^{1-0}(\operatorname{Dom}(T))$;

$\left(P_{5}\right) A_{*} \in C^{1-0}\left(\operatorname{Dom}\left(A_{*}\right)\right)$ and there exist three positive constants $\delta_{0}, C_{0}$ and $t_{0}$ with $\delta_{0}<\frac{1}{n}, 0<t_{0}<\min _{x \in \bar{\Omega}} T(x)$ such that

$$
\left|\nabla_{x} A_{*}(x, t)\right| \leq C_{0}\left(A_{*}(x, t)\right)^{1+\delta_{0}}, \quad j=1, \ldots, n,
$$

for $x \in \Omega$ and $|t| \in\left[t_{0}, T(x)\right)$ provided $\nabla_{x} A_{*}(x, t)$ exists.

Let $A, B \in N(\Omega)$. We say that $A \ll B$ if, for any $k>0$,

$$
\lim _{t \rightarrow+\infty} \frac{A(x, k t)}{B(x, t)}=0 \text { uniformly for } x \in \Omega \text {. }
$$

Remark 2.2. Suppose that $A, B \in N(\Omega)$, then $A \ll B \Rightarrow A \preccurlyeq B$.

Next we give two embedding theorems for Musielak-Orlicz-Sobolev spaces recently developed by Fan in [18].

Theorem 2.7. (See [18, 28]) Let $\left(P_{1}\right)-\left(P_{5}\right)$ hold. Then

(i) There is a continuous imbedding $W^{1, A}(\Omega) \hookrightarrow L^{A_{*}}(\Omega)$;

(ii) Suppose that $B \in N(\Omega), B: \bar{\Omega} \times[0,+\infty) \rightarrow[0,+\infty)$ is continuous, and $B(x, t) \in(0,+\infty)$ for $x \in \Omega$ and $t \in(0,+\infty)$. If $B \ll A_{*}$, then there is a compact imbedding $W^{1, A}(\Omega) \hookrightarrow \hookrightarrow L^{B}(\Omega)$.

By Theorem 2.7. Remark 2.2 and Proposition 2.6, we have the following:

Theorem 2.8. (See [18, 28]) Let $\left(P_{1}\right)-\left(P_{5}\right)$ hold and furthermore, $A, A_{*} \in N(\Omega)$. Then

(i) $A \ll A_{*}$, and there is a compact imbedding $W^{1, A}(\Omega) \hookrightarrow \hookrightarrow L^{A}(\Omega)$; 
HÖLDER CONTINUITY FOR NONLINEAR ELLIPTIC PROBLEM IN MUSIELAK-ORLICZ-SOBOLEV SPACE *

(ii) there holds the poincaré-type inequality

$$
\|u\|_{A} \leq C\|\nabla u\|_{A} \text { for } u \in W_{0}^{1, A}(\Omega),
$$

i.e. $\|\nabla u\|_{A}$ is an equivalent norm on $W_{0}^{1, A}(\Omega)$.

\section{Some Lemmas}

Suppose $\Omega \subset \mathbb{R}^{n}$ is a bounded smooth domain, and $A \in N(\Omega)$ satisfies the following Condition $(\mathscr{A})$, denoted by $A \in \mathscr{A}$.

$(\mathscr{A}) A \in N(\Omega)$ satisfies assumptions $\left(P_{1}\right),\left(P_{2}\right),\left(P_{3}\right),\left(P_{5}\right)$ in Section 2 and the following

$\left(\widetilde{P_{4}}\right) T(x)$ defined in (2.4) satisfies $T(x)=+\infty$ for all $x \in \bar{\Omega}$.

Lemma 3.1. (see [?]) Suppose that $A \in N(\Omega)$, and there exists a strictly increasing differentiable function $\mathfrak{A}:[0,+\infty) \rightarrow[0,+\infty)$ such that

$$
A(x, \alpha t) \geq \mathfrak{A}(\alpha) A(x, t), \forall \alpha \geq 0, t \in \mathbb{R}, x \in \Omega .
$$

(i) Then there exists a strictly increasing differentiable function $\widehat{\mathfrak{A}}:[0,+\infty) \rightarrow$ $[0,+\infty)$, defined by

$$
\widehat{\mathfrak{A}}(\beta)= \begin{cases}\frac{1}{\mathfrak{A}\left(\frac{1}{\beta}\right)}, & \text { for } \beta>0, \\ 0, & \text { for } \beta=0,\end{cases}
$$

such that

$$
A(x, \beta t) \leq \widehat{\mathfrak{A}}(\beta) A(x, t), \forall \beta>0, t \in \mathbb{R}, x \in \Omega,
$$

and furthermore $\widehat{\widehat{A}}=\mathfrak{A}$;

(ii) If $\mathfrak{A}$ satisfies

$$
n \mathfrak{A}(\alpha)>\alpha \mathfrak{A}^{\prime}(\alpha),
$$

then $A_{*} \in N(\Omega)$, and there exists a strictly increasing differentiable function $\mathfrak{A}_{*}:[0,+\infty) \rightarrow[0,+\infty)$, defined by

such that

$$
\mathfrak{A}_{*}^{-1}(\sigma)= \begin{cases}\frac{1}{\sigma^{\frac{1}{n} \mathfrak{A}^{-1}\left(\sigma^{-1}\right)},}, & \text { for } \sigma>0, \\ 0, & \text { for } \sigma=0,\end{cases}
$$

$$
A_{*}(x, \beta t) \leq \mathfrak{A}_{*}(\beta) A_{*}(x, t), \forall \beta>0, t \in \mathbb{R}, x \in \Omega ;
$$

(iii) If $\mathfrak{A}$ satisfies

$$
\alpha \mathfrak{A}^{\prime}(\alpha)>\mathfrak{A}(\alpha)
$$

then $\widetilde{A} \in N(\Omega)$, and there exists two strictly increasing differentiable functions $\widetilde{\mathfrak{A}}, \widehat{\widetilde{\mathfrak{A}}}:[0,+\infty) \rightarrow[0,+\infty)$, defined by

$$
\widetilde{\mathfrak{A}}^{-1}(\sigma)= \begin{cases}\frac{\sigma}{\mathfrak{A}^{-1}(\sigma)}, & \text { for } \sigma>0, \\ 0, & \text { for } \sigma=0,\end{cases}
$$

and

$$
\widehat{\widetilde{\mathfrak{A}}}^{-1}(\sigma)= \begin{cases}\sigma \mathfrak{A}^{-1}\left(\sigma^{-1}\right), & \text { for } \sigma>0 \\ 0, & \text { for } \sigma=0\end{cases}
$$


such that

$$
\begin{aligned}
& \widetilde{A}(x, \beta t) \leq \widetilde{\mathfrak{A}}(\beta) \widetilde{A}(x, t), \forall \beta>0, t \in \mathbb{R}, x \in \Omega . \\
& \widetilde{A}(x, \beta t) \geq \widehat{\widetilde{\mathfrak{A}}}(\beta) \widetilde{A}(x, t), \forall \beta>0, t \in \mathbb{R}, x \in \Omega .
\end{aligned}
$$

Definition 3.1. We say that $\mathfrak{C}: \mathbb{R}^{+} \rightarrow \mathbb{R}^{+}$satisfies Condition $\Delta_{\mathbb{R}^{+}}$, denoted by $\mathfrak{C} \in \Delta_{\mathbb{R}^{+}}$, if there exists a constant $M_{0}>0$ such that

$$
\mathfrak{C}(\alpha \beta) \leq M_{0} \mathfrak{C}(\alpha) \mathfrak{C}(\beta), \forall \alpha, \beta>0 .
$$

Remark 3.1. By (3.2), (3.5) and (3.8), if $\mathfrak{A}, \mathfrak{A}^{-1} \in \Delta_{\mathbb{R}^{+}}$, then $\widehat{\mathfrak{A}}, \mathfrak{A}_{*}^{-1}, \widetilde{\mathfrak{A}}^{-1}, \widehat{\widetilde{\mathfrak{A}}}^{-1} \in$ $\Delta_{\mathbb{R}^{+}}$, and there exists a constant $M_{0}>0$ such that the following two inequalities hold

$$
\mathfrak{A}^{-1}(\alpha) \mathfrak{A}^{-1}\left(\alpha^{-1}\right) \leq M_{0}, \quad \alpha \mathfrak{A}^{-1}\left(\widehat{\mathfrak{A}}\left(\alpha^{-1}\right)\right) \leq M_{0}, \forall \alpha>0 .
$$

Lemma 3.2. (see [?]) Let $\left\{y_{h}\right\} \subset \mathbb{R}$ be a sequence satisfying

$$
y_{h+1} \leq \frac{1}{\beta} \mathfrak{A}_{*}\left(\frac{\mathfrak{A}^{-1}(\beta)}{\beta^{\frac{1}{n}}} c 2^{h} \mathfrak{A}^{-1}\left(c \mathfrak{A}_{*}\left(2^{h+2}\right) y_{h}\right)\right), \forall \beta>0,
$$

where $c$ is a positive constant. If $\mathfrak{A}, \mathfrak{A}^{-1}, \mathfrak{A}_{*} \in \Delta_{\mathbb{R}^{+}}$then there exists a $y_{0}^{*}>0$ such that for $y_{0} \leq y_{0}^{*}, y_{h} \rightarrow 0$ as $h \rightarrow \infty$.

For a measurable set $E \subset \mathbb{R}^{n}$, we denote by $\operatorname{mes}(E)$ or $|E|$ the $n$-Lebesgue measure of $E$. For a measurable function $u$ defined in $\Omega$ and a measurable set $E \subset \Omega$ denote

$$
\begin{aligned}
& \max _{E} u(x):=\operatorname{ess}_{\sup _{x \in E}} u(x), \quad \min _{E} u(x):=\operatorname{ess}_{\inf } \operatorname{seE}_{x \in E} u(x), \\
& \operatorname{osc}(u ; E):=\max _{E} u(x)-\min _{E} u(x) .
\end{aligned}
$$

If $u \in W^{1, A}(\Omega)$ and $B_{\rho}=B_{\rho}(x):=\left\{y \in \mathbb{R}^{n}:|y-x|<\rho\right\} \subset \Omega$ is any given ball, we denote $\Omega_{k, \rho}:=\left\{x \in B_{\rho}: u(x)>k\right\}$, where $k$ is a real number.

The following two lemmas will be used.

Lemma 3.3. (see Lemma 3.9 of Chapter 2 of [23]) For any $u \in W^{1,1}\left(B_{\rho}\right)$ and arbitrary number $k$ and $l$ with $l>k$, the following inequality holds

$$
(l-k)\left|\Omega_{l, \rho}\right|^{1-\frac{1}{n}} \leq C \frac{\rho^{n}}{\left|B_{\rho}-\Omega_{k, \rho}\right|} \int_{\Omega_{k, \rho} \backslash \Omega_{l, \rho}}|\nabla u| \mathrm{d} x,
$$

where $C=C(n)>1$ is a constant depending only on $n$.

Lemma 3.4. (see Lemma 4.8 of Chapter 2 of 23) Suppose a function $u(x)$ is measurable and bounded in some ball $B_{R_{0}}$. Consider balls $B_{R}$ and $B_{b R}$ which have a common center with $B_{R_{0}}$, where $b>1$ is a fixed constant. Suppose in addition that for any $0<R \leq b^{-1} R_{0}$ at least one of the following two inequalities is valid

$$
\operatorname{osc}\left\{u ; B_{R}\right\} \leq c_{1} R^{\varepsilon}, \quad \operatorname{osc}\left\{u, B_{R}\right\} \leq \theta \operatorname{osc}\left\{u ; B_{b R}\right\},
$$


where $c_{1}>0, \varepsilon \leq 1$ and $\theta<1$ are positive constants. Then $u \in C^{0, \alpha}\left(B_{R_{0}}\right)$, where $\alpha=\min \left\{\varepsilon,-\log _{b} \theta\right\}$.

In the following lemma $A \in N(\Omega) \cap \mathscr{A}$ satisfies the following assumptions:

$\left(A_{1}\right)$ There exists a strictly increasing differentiable function $\mathfrak{A}:[0,+\infty) \rightarrow$ $[0,+\infty)$ satisfying

$$
n \mathfrak{A}(\alpha)>\alpha \mathfrak{A}^{\prime}(\alpha)>\mathfrak{A}(\alpha)
$$

such that

$\left(A_{11}\right) A(x, \alpha t) \geq \mathfrak{A}(\alpha) A(x, t), \forall \alpha \geq 0, t \in \mathbb{R}, x \in \Omega$;

$\left(A_{12}\right) \mathfrak{A}, \mathfrak{A}^{-1}, \mathfrak{A}_{*}, \widehat{\widetilde{\mathfrak{A}}}, \in \Delta_{\mathbb{R}^{+}}$;

Lemma 3.5. (see [?]) Let $A \in N(\Omega) \cap \mathscr{A}$ satisfy $\left(A_{1}\right), B \in N(\Omega)$ satisfy $B \preccurlyeq A_{*}$, and $u \in W^{1, A}(\Omega)$ satisfy for any $B_{R} \subset \Omega, R \leq R_{0}$ and for all $\sigma \in(0,1)$ and any $k \geq t_{0}$,

$$
\int_{\Omega_{k, \sigma R}} A(x,|\nabla u|) \mathrm{d} x \leq \gamma \int_{\Omega_{k, R}} A_{*}\left(x, \frac{u-k}{(1-\sigma) R}\right) \mathrm{d} x+\gamma_{1} \int_{\Omega_{k, R}} B(x, k) \mathrm{d} x .
$$

Then $u$ is locally bounded above in $\Omega$.

Definition 3.2. Let $M, \gamma, \gamma_{1}$ and $\delta$ are positive constants with $\delta \leq 2$. We will say that a function $u$ belongs to class $\mathscr{B}\left(\Omega, M, \gamma, \gamma_{1}, \delta\right)$ if $u \in W^{1, A}(\Omega), \max _{\Omega}|u(x)| \leq$ $M$ and the functions $w(x)= \pm u(x)$ satisfy the inequality

$$
\int_{\Omega_{k, \sigma \rho}} A(x,|\nabla w|) \mathrm{d} x \leq \gamma \int_{\Omega_{k, \rho}} A\left(x, \frac{w(x)-k}{(1-\sigma) \rho}\right) \mathrm{d} x+\gamma_{1}\left|\Omega_{k, \rho}\right|
$$

for arbitrary $B_{\rho} \subset \Omega, \sigma \in(0,1)$ and such $k$

$$
k \geq \max _{B_{\rho}} w(x)-\delta M .
$$

The main result of this section is the following:

Theorem 3.1. Let $A \in N(\Omega) \cap \mathscr{A}$ satisfy $\left(A_{1}\right)$ and $\left(C_{1}\right)$. Then $\mathscr{B}\left(\Omega, M, \gamma, \gamma_{1}, \delta\right) \subset$ $C^{0, \alpha}(\Omega)$, where the constant $\alpha \in(0,1)$ depends only on the parameters $n, A, \gamma$ and $\delta$, but it is independent of $\gamma_{1}$ and $M$.

We are now on the position to prove Theorem 3.1. Let $u \in \mathscr{B}\left(\Omega, M, \gamma, \gamma_{1}, \delta\right)$ in which $A$ satisfies the condition $\left(A_{1}\right)$ and $\left(C_{1}\right)$. Without loss of generality we may assume that $M \geq 1$ and $\gamma \geq 1$. To prove Theorem 3.1 it suffices to prove that for each $x_{0} \in \Omega$ there is a ball $B_{R_{0}} \subset \Omega$ such that $u \in C^{0, \alpha}\left(B_{R_{0}}\right)$ where $\alpha=\alpha(n, A, \gamma, \delta)$ is a constant.

Now let $x_{0} \in \Omega$ be given arbitrarily. Choose a positive number $R_{0}<1$ such that $\overline{B_{R_{0}}\left(x_{0}\right)} \subset \Omega$, take arbitrarily $R \in\left(0, R_{0}\right]$. It is easy to see that at least one of the two functions $w= \pm u$ satisfies the following condition

$$
\operatorname{mes}\left\{x \in B_{\frac{R}{2}}: w(x)>\max _{B_{R}} w(x)-\frac{1}{2} \operatorname{osc}\left\{u ; B_{R}\right\}\right\} \leq \frac{1}{2} \operatorname{mes} B_{\frac{R}{2}} .
$$


From now on we denote by $w$ the function identified to $u$ or $-u$ that satisfies (3.18). Set

$$
\tau=\max \{2,2 / \delta\}, \quad \psi=\tau^{-1} \operatorname{osc}\left\{u ; B_{R}\right\}, \quad k^{\prime}=\max _{B_{R}} w(x)-\psi .
$$

Then

$$
k^{\prime} \geq \max _{B_{R}} w(x)-\frac{1}{2} \operatorname{osc}\left\{u ; B_{R}\right\}
$$

and

$$
k^{\prime} \geq \max _{B_{R}} w(x)-\delta M .
$$

Note that (3.21) implies that (3.16) and (3.17) hold for $k \geq k^{\prime}$ and $\rho \leq R$.

Under the above assumptions we can give the proof of Theorem 3.1 throughout the following Lemmas 3.6. 3.9.

Lemma 3.6. Let $A \in N(\Omega) \cap \mathscr{A}$ satisfy $\left(A_{1}\right)$ and $\left(C_{1}\right)$. Then there is a positive constant $\theta=\theta(n, A, \gamma)$ such that the following equation

$$
\left|\Omega_{k^{0}, \frac{R}{2}}\right| \leq \theta R^{n},
$$

implies that at least one of the following two inequalities holds:

$$
\begin{gathered}
H \leq \widehat{\mathfrak{A}}^{-1}\left(\frac{\gamma_{1}}{\gamma}\right) R, \\
\max _{B_{R / 4}} w(x) \leq k^{0}+\frac{H}{2},
\end{gathered}
$$

where

$$
0<H<\psi, k^{0}=\max _{B_{R}} w(x)-H
$$

Proof. Set

$$
t=A^{-1}\left(x, \frac{s}{\mathfrak{A}(\alpha)}\right)
$$

in (3.1). Then for $\alpha>0$ we have

$$
A\left(x, \alpha A^{-1}\left(x, \frac{s}{\mathfrak{A}(\alpha)}\right)\right) \geq \mathfrak{A}(\alpha) A\left(x, A^{-1}\left(x, \frac{s}{\mathfrak{A}(\alpha)}\right)\right)=s,
$$

or equivalently

which implies that

$$
\alpha A^{-1}\left(x, \frac{s}{\mathfrak{A}(\alpha)}\right) \geq A^{-1}(x, s)
$$

$$
\frac{\alpha}{(\mathfrak{A}(\alpha))^{\frac{1}{n}}} \frac{A^{-1}\left(x, \frac{s}{\mathfrak{A}(\alpha)}\right)}{\left(\frac{s}{\mathfrak{A}(\alpha)}\right)^{\frac{n+1}{n}}} \frac{1}{\mathfrak{A}(\alpha)} \geq \frac{A^{-1}(x, s)}{s^{\frac{n+1}{n}}} .
$$

Integrating the above inequality with respect to $s$ from 0 to $t$, we have

$$
\frac{\alpha}{(\mathfrak{A}(\alpha))^{\frac{1}{n}}} \int_{0}^{\frac{t}{\mathfrak{2}(\alpha)}} \frac{A^{-1}(x, r)}{r^{\frac{n+1}{n}}} \mathrm{~d} r \geq \int_{0}^{t} \frac{A^{-1}(x, s)}{s^{\frac{n+1}{n}}} \mathrm{~d} s,
$$


HÖLDER CONTINUITY FOR NONLINEAR ELLIPTIC PROBLEM IN MUSIELAK-ORLICZ-SOBOLEV SPACE where $r=\frac{s}{\mathfrak{A}(\alpha)}$. Then the definition of $A_{*}$ in $(2.3)$ yields that

$$
\frac{\alpha}{(\mathfrak{A}(\alpha))^{\frac{1}{n}}} A_{*}^{-1}\left(x, \frac{t}{\mathfrak{A}(\alpha)}\right) \geq A_{*}^{-1}(x, t) .
$$

Setting $t=\mathfrak{A}(\alpha) A_{*}\left(x, \frac{\mathfrak{A}(\alpha)}{\alpha}\right)$ in $(3.26)$, we conclude that

$$
(\mathfrak{A}(\alpha))^{1-\frac{1}{n}} \geq A_{*}^{-1}\left(x, \mathfrak{A}(\alpha) A_{*}\left(x, \frac{\mathfrak{A}(\alpha)}{\alpha}\right)\right),
$$

or equivalently

$$
A_{*}\left(x, \mu^{1-\frac{1}{n}}\right) \geq \mu A_{*}\left(x, \frac{\mu}{\mathfrak{A}^{-1}(\mu)}\right),
$$

in which $\mu=\mathfrak{A}(\alpha)$. Then by (3.6) and the above (3.27), we have

$$
A_{*}\left(x, \mu^{1-\frac{1}{n}}\right) \geq \mu A_{*}\left(x, \frac{\mu}{\mathfrak{A}^{-1}(\mu)}\right) \geq A_{*}\left(x, \mathfrak{A}_{*}^{-1}(\mu) \frac{\mu}{\mathfrak{A}^{-1}(\mu)}\right),
$$

which implies that

$$
\mathfrak{A}_{*}^{-1}(\mu) \mu \leq \mu^{1-\frac{1}{n}} \mathfrak{A}^{-1}(\mu)
$$

For $h=0,1,2 \cdots$, set

$$
\begin{aligned}
& \rho_{h}=\frac{R}{4}+\frac{R}{2^{h+2}}, \quad k_{h}=k^{0}+\frac{H}{2}-\frac{H}{2^{h+1}}, \\
& y_{h}=R^{-n}\left|\Omega_{k_{h}, \rho_{h}}\right|, \quad D_{h+1}=\Omega_{k_{h}, \rho_{h+1}} \backslash \Omega_{k_{h+1}, \rho_{h+1}} .
\end{aligned}
$$

Applying inequality (3.13) to $l=k_{h+1}, k=k_{h}$ and $\rho=\rho_{h+1}$ we get

$$
\begin{aligned}
& \begin{array}{l}
1-\frac{1}{n} \\
y_{h+1}
\end{array} \\
& =R^{1-n}\left|\Omega_{k_{h+1}, \rho_{h+1}}\right|^{1-\frac{1}{n}} \\
& \leq \frac{C R^{1-n}}{\left(k_{h+1}-k_{h}\right)} \frac{\rho_{h+1}^{n}}{\left|B_{\rho_{h+1}}-\Omega_{k_{h}, \rho_{h+1}}\right|} \int_{D_{h+1}}|\nabla w| \mathrm{d} x \\
& \leq C R^{1-n} 2^{h+2} H^{-1}\left(\frac{R}{2}\right)^{n} \frac{1}{\left|B_{R / 4}-\Omega_{k^{0}, \frac{R}{2}}\right|} 2|\nabla w|_{A ; D_{h+1}} \cdot|1|_{\widetilde{A} ; D_{h+1}} \\
& =\frac{C 2^{h+3-n} H^{-1} R}{\left|B_{R / 4}-\Omega_{k^{0}, \frac{R}{2}}\right|} \mathfrak{A}^{-1}\left(\mathfrak{A}\left(|\nabla w|_{A ; D_{h+1}}\right) \int_{D_{h+1}} A\left(x, \frac{|\nabla w|}{|\nabla w|_{A ; D_{h+1}}}\right) \mathrm{d} x\right) \\
& \cdot \widehat{\widetilde{\mathfrak{A}}}^{-1}\left(\widehat{\widetilde{\mathfrak{A}}}\left(|1|_{\widetilde{A} ; D_{h+1}}\right) \cdot \int_{D_{h+1}} \widetilde{A}\left(x, \frac{1}{|1|_{\widetilde{A} ; D_{h+1}}}\right) \mathrm{d} x\right) \\
& \leq \frac{C 2^{h+3-n} H^{-1} R}{\left|B_{R / 4}-\Omega_{k^{0}, \frac{R}{2}}\right|} \mathfrak{A}^{-1}\left(\int_{D_{h+1}} A(x,|\nabla w|) \mathrm{d} x\right) \cdot \widehat{\widetilde{\mathfrak{A}}}^{-1}\left(\int_{D_{h+1}} \widetilde{A}(x, 1) \mathrm{d} x\right) \\
& \leq \frac{C 2^{h+3-n} H^{-1} R}{\left|B_{R / 4}-\Omega_{k^{0}, \frac{R}{2}}\right|} \mathfrak{A}^{-1}\left(\int_{D_{h+1}} A(x,|\nabla w|) \mathrm{d} x\right) \cdot \widehat{\widetilde{\mathfrak{A}}}^{-1}\left(c_{1}\left|D_{h+1}\right|\right) \\
& \leq \frac{C 2^{h+3-n} H^{-1} R}{\left|B_{R / 4}-\Omega_{k^{0}, \frac{R}{2}}\right|} \mathfrak{A}^{-1}\left(\int_{D_{h+1}} A(x,|\nabla w|) \mathrm{d} x\right) \cdot \widehat{\widetilde{\mathfrak{A}}}^{-1}\left(c_{2} R^{n} y_{h}\right) .
\end{aligned}
$$


If (3.22) holds, choose

$$
\theta \leq \frac{1}{2} \cdot 4^{-n} \omega_{n}
$$

Then

$$
\left|B_{R / 4}-\Omega_{k^{0}, \frac{R}{2}}\right| \geq 4^{-n} R^{n} \omega_{n}-\frac{1}{2} \cdot 4^{-n} \omega_{n} R^{n}=2^{-2 n-1} \omega_{n} R^{n},
$$

and consequently from (3.29) it follows that

$$
y_{h+1}^{1-\frac{1}{n}} \leq c 2^{h} R^{1-n} H^{-1} \omega_{n}^{-1} \mathfrak{A}^{-1}\left(\int_{D_{h+1}} A(x,|\nabla w|) \mathrm{d} x\right) \widehat{\widetilde{\mathfrak{A}}}^{-1}\left(c_{2} R^{n} y_{h}\right) .
$$

Applying inequality (3.16) with $k=k_{h}, \rho=\rho_{h}, \sigma \rho=\rho_{h+1}$, we obtain

$$
\begin{aligned}
& \int_{D_{h+1}} A(x,|\nabla w|) \mathrm{d} x \\
\leq & \int_{\Omega_{k_{h}, \rho_{h+1}}} A(x,|\nabla w|) \mathrm{d} x \\
\leq & \int_{\Omega_{k_{h}, \rho_{h}}} A\left(x, \frac{w-k_{h}}{\rho_{h}-\rho_{h+1}}\right) \mathrm{d} x+\gamma_{1}\left|\Omega_{k_{h}, \rho_{h}}\right| \\
\leq & \int_{\Omega_{k_{h}, \rho_{h}}} A\left(x, \frac{2^{h+3}}{R}\left|w-k_{h}\right|\right) \mathrm{d} x+\gamma_{1}\left|\Omega_{k_{h}, \rho_{h}}\right| \\
\leq & \int_{\Omega_{k_{h}, \rho_{h}}} A\left(x, \frac{2^{h+3}}{R} H\right) \mathrm{d} x+\gamma\left|\Omega_{k_{h}, \rho_{h}}\right| \\
\leq & \gamma \widehat{\mathfrak{A}}\left(R^{-1} H\right) \int_{\Omega_{k_{h}, \rho_{h}}} A\left(x, 2^{h+3}\right) \mathrm{d} x+\gamma_{1}\left|\Omega_{k_{h}, \rho_{h}}\right| \\
\leq & \gamma \widehat{\mathfrak{A}}\left(R^{-1} H\right)\left[\int_{\Omega_{k_{h}, \rho_{h}}} A_{*}\left(x, 2^{h+3}\right) \mathrm{d} x+\int_{\Omega_{k_{h}, \rho_{h}}} C_{1} \mathrm{~d} x\right]+\gamma_{1}\left|\Omega_{k_{h}, \rho_{h}}\right| \\
\leq & \gamma \widehat{\mathfrak{A}}\left(R^{-1} H\right)\left[C_{2} \mathfrak{A}_{*}\left(2^{h+2}\right)\left|\Omega_{k_{h}, \rho_{h}}\right|+C_{1}\left|\Omega_{k_{h}, \rho_{h}}\right|\right]+\gamma_{1}\left|\Omega_{k_{h}, \rho_{h}}\right| \\
\leq & C \gamma \widehat{\mathfrak{A}}\left(R^{-1} H\right) \mathfrak{A}_{*}\left(2^{h+2}\right)\left|\Omega_{k_{h}, \rho_{h}}\right|+\gamma_{1}\left|\Omega_{k_{h}, \rho_{h}}\right| .
\end{aligned}
$$

Assume that equation (3.23) does not hold, then we have

$$
\int_{D_{h+1}} A(x,|\nabla w|) \mathrm{d} x \leq c \gamma \widehat{\mathfrak{A}}\left(R^{-1} H\right) \mathfrak{A}_{*}\left(2^{h+2}\right) R^{n} y_{h}
$$

Then from (3.30), we can get

$$
y_{h+1}^{1-\frac{1}{n}} \leq c 2^{h} R^{1-n} H^{-1} \omega_{n}^{-1} \mathfrak{A}^{-1}\left(c \gamma \widehat{\mathfrak{A}}\left(R^{-1} H\right) \mathfrak{A}_{*}\left(2^{h+2}\right) R^{n} y_{h}\right) \widehat{\widetilde{\mathfrak{A}}}^{-1}\left(c_{2} R^{n} y_{h}\right) .
$$

For any $\beta>0$, set $\mu=\beta y_{h+1}$ in (3.28). Then

$$
\mathfrak{A}_{*}^{-1}\left(\beta y_{h+1}\right) \beta y_{h+1} \leq \beta^{1-\frac{1}{n}} y_{h+1}^{1-\frac{1}{n}} \mathfrak{A}^{-1}\left(\beta y_{h+1}\right),
$$

or equivalently

$$
\mathfrak{A}_{*}^{-1}\left(\beta y_{h+1}\right) y_{h+1} \leq \frac{1}{\beta^{\frac{1}{n}}} y_{h+1}^{1-\frac{1}{n}} \mathfrak{A}^{-1}\left(\beta y_{h+1}\right) .
$$

By the definition of $y_{h}$, it is clear that $y_{h} \downarrow \varepsilon_{0} \geq 0$ as $h \rightarrow \infty$.

Claim: if (3.23) does not hold, then $\varepsilon_{0}=0$. 
HÖLDER CONTINUITY FOR NONLINEAR ELLIPTIC PROBLEM IN MUSIELAK-ORLICZ-SOBOLEV SPACE 4 本

To prove $\varepsilon_{0}=0$, we argue by the contrary $\varepsilon_{0}>0$. Then Lemma 3.1 Remark 3.1, $\mathfrak{A}^{-1}, \mathfrak{A} \in \Delta_{\mathbb{R}^{+}},(3.33)$ and (3.34) imply

$$
\begin{aligned}
& \mathfrak{A}_{*}^{-1}\left(\beta y_{h+1}\right) y_{h+1} \\
& \leq \frac{1}{\beta^{\frac{1}{n}}} y_{h+1}^{1-\frac{1}{n}} \mathfrak{A}^{-1}\left(\beta y_{h+1}\right) \\
& \leq \frac{\mathfrak{A}^{-1}(\beta)}{\beta^{\frac{1}{n}}} c y_{h+1}^{1-\frac{1}{n}} \mathfrak{A}^{-1}\left(y_{h+1}\right) \\
& \leq \frac{\mathfrak{A}^{-1}(\beta)}{\beta^{\frac{1}{n}}} c 2^{h} R^{1-n} H^{-1} \omega_{n}^{-1} \mathfrak{A}^{-1}\left(c \gamma \widehat{\mathfrak{A}}\left(R^{-1} H\right) \mathfrak{A}_{*}\left(2^{h+2}\right) R^{n} y_{h}\right) \\
& \widehat{\widetilde{\mathfrak{A}}}^{-1}\left(c_{2} R^{n} y_{h}\right) \mathfrak{A}^{-1}\left(y_{h+1}\right) \\
& =\frac{\mathfrak{A}^{-1}(\beta)}{\beta^{\frac{1}{n}}} c 2^{h} R^{1-n} H^{-1} \omega_{n}^{-1} \mathfrak{A}^{-1}\left(c \gamma \widehat{\mathfrak{A}}\left(R^{-1} H\right) \mathfrak{A}_{*}\left(2^{h+2}\right) R^{n} y_{h}\right) \\
& \cdot c_{2} R^{n} y_{h} \mathfrak{A}^{-1}\left(\frac{1}{c_{2} R^{n} y_{h}}\right) \mathfrak{A}^{-1}\left(y_{h+1}\right) \\
& \leq \frac{\mathfrak{A}^{-1}(\beta)}{\beta^{\frac{1}{n}}} c 2^{h} R H^{-1} \omega_{n}^{-1} \mathfrak{A}^{-1}\left(c \gamma \widehat{\mathfrak{A}}\left(R^{-1} H\right) \mathfrak{A}_{*}\left(2^{h+2}\right) R^{n} y_{h}\right) \cdot c_{3} y_{h} \mathfrak{A}^{-1}\left(\frac{y_{h+1}}{c_{2} R^{n} y_{h}}\right) \\
& \leq \frac{\mathfrak{A}^{-1}(\beta)}{\beta^{\frac{1}{n}}} c 2^{h} R H^{-1} \omega_{n}^{-1} \mathfrak{A}^{-1}\left(c \gamma \widehat{\mathfrak{A}}\left(R^{-1} H\right) \mathfrak{A}_{*}\left(2^{h+2}\right) R^{n} y_{h}\right) \cdot c_{4} y_{h} \mathfrak{A}^{-1}\left(R^{-n}\right) \\
& \leq \frac{\mathfrak{A}^{-1}(\beta)}{\beta^{\frac{1}{n}}} c_{5} 2^{h} \omega_{n}^{-1} \mathfrak{A}^{-1}\left(c \gamma \mathfrak{A}_{*}\left(2^{h+2}\right) y_{h}\right) \cdot y_{h} \mathfrak{A}^{-1}\left(R^{n}\right) \mathfrak{A}^{-1}\left(R^{-n}\right) \\
& \cdot\left(R H^{-1}\right) \mathfrak{A}^{-1}\left(\widehat{\mathfrak{A}}\left(R^{-1} H\right)\right) \\
& \leq \frac{\mathfrak{A}^{-1}(\beta)}{\beta^{\frac{1}{n}}} c_{5} 2^{h} \omega_{n}^{-1} \mathfrak{A}^{-1}\left(c \gamma \mathfrak{A}_{*}\left(2^{h+2}\right) y_{h}\right) \cdot y_{h} \cdot M_{0}^{2},
\end{aligned}
$$

and therefore

$$
\begin{aligned}
\mathfrak{A}_{*}^{-1}\left(\beta y_{h+1}\right) & \leq \frac{\mathfrak{A}^{-1}(\beta)}{\beta^{\frac{1}{n}}} c_{6} 2^{h} \omega_{n}^{-1} \mathfrak{A}^{-1}\left(c \gamma \mathfrak{A}_{*}\left(2^{h+2}\right) y_{h}\right) \cdot \frac{y_{h}}{y_{h+1}} \\
& \leq \frac{\mathfrak{A}^{-1}(\beta)}{\beta^{\frac{1}{n}}} c 2^{h} \mathfrak{A}^{-1}\left(c \mathfrak{A}_{*}\left(2^{h+2}\right) y_{h}\right) .
\end{aligned}
$$

Then from the above inequality we conclude

$$
y_{h+1} \leq \frac{1}{\beta} \mathfrak{A}_{*}\left(\frac{\mathfrak{A}^{-1}(\beta)}{\beta^{\frac{1}{n}}} c 2^{h} \mathfrak{A}^{-1}\left(c \mathfrak{A}_{*}\left(2^{h+2}\right) y_{h}\right)\right), \forall \beta>0 .
$$

Choose $\theta \leq \min \left\{y_{0}^{*}, \frac{1}{2} \cdot 4^{-n} \omega_{n}\right\}$. Then (3.22) yields that $y_{0} \leq \theta \leq y_{0}^{*}$. By Lemma 3.2 we can get $y_{h} \downarrow 0=\varepsilon_{0}$ as $h \rightarrow \infty$, which contradicts to $\varepsilon_{0}>0$.

The conclusion of the Claim means that (3.24) holds. The proof is completed.

Lemma 3.7. Let $A \in N(\Omega) \cap \mathscr{A}$ satisfy $\left(A_{1}\right)$ and $\left(C_{1}\right)$. For any given $\theta>0$ there is a natural number $s=s(\theta, n, A, \gamma)>2$ such that either

$$
\psi \leq 2^{s} \widehat{\mathfrak{A}}^{-1}\left(\frac{\gamma_{1}}{\gamma}\right) R
$$


or

$$
\left|\Omega_{k^{0}, \frac{R}{2}}\right| \leq \theta R^{n}
$$

holds, where $k^{0}=\max _{B_{R}} w(x)-2^{-s+1} \psi$.

Proof. If equation (3.36) does not hold, i.e.

$$
\psi>2^{s \widehat{\mathfrak{A}}^{-1}}\left(\frac{\gamma_{1}}{\gamma}\right) R
$$

where the nature number s will be determined later.

For $t=0,1,2 \ldots, s-1$, set

$$
k_{t}=\max _{B_{R}} w(x)-2^{-t} \psi, \quad D_{t}=\Omega_{k_{t}, \frac{R}{2}} \backslash \Omega_{k_{t+1}, \frac{R}{2}} .
$$

Applying inequality (3.16) with $\rho=R, \rho-\sigma \rho=\frac{R}{2}, k=k_{t}$ for $t=0,1,2, \ldots, s-2$, and by (3.38) we obtain

$$
\begin{aligned}
& \int_{D_{t}} A(x,|\nabla w|) \mathrm{d} x \\
\leq & \int_{\Omega_{k_{t}, \frac{R}{2}}} A(x,|\nabla w|) \mathrm{d} x \\
\leq & \gamma \int_{\Omega_{k_{t}, R}} A\left(x, \frac{w-k_{t}}{\frac{R}{2}}\right) \mathrm{d} x+\gamma_{1}\left|\Omega_{k_{t}, R}\right| \\
\leq & \gamma \int_{\Omega_{k_{t}, R}} A\left(x, \frac{2}{R} 2^{-t} \psi\right) \mathrm{d} x+\gamma_{1}\left|\Omega_{k_{t}, R}\right| \\
\leq & C_{1} \widehat{\mathfrak{A}}\left(R^{-1} 2^{-t} \psi\right)\left|\Omega_{k_{t}, R}\right|+\gamma_{1}\left|\Omega_{k_{t}, R}\right| \\
\leq & c \gamma \widehat{\mathfrak{A}}\left(R^{-1} 2^{-t} \psi\right) R^{n} \quad(t=0,1, \cdots, s-2) .
\end{aligned}
$$

Applying inequality (3.13) to $l=k_{t+1}, k=k_{t}$ and $\rho=\frac{R}{2}$ we get $(3.40)$

$$
\begin{aligned}
& \left|\Omega_{k_{s-1}, \frac{R}{2}}\right|^{1-\frac{1}{n}} \\
\leq & \left|\Omega_{k_{t+1}, \frac{R}{2}}\right|^{1-\frac{1}{n}} \\
\leq & \frac{C}{\left(k_{t+1}-k_{t}\right)} \frac{\left(\frac{R}{2}\right)^{n}}{\left|B_{\frac{R}{2}}-\Omega_{k_{t}, \frac{R}{2}}\right|} \int_{D_{t}}|\nabla w| \mathrm{d} x \\
\leq & C\left(2^{-(t+1)} \psi\right)^{-1}\left(\frac{R}{2}\right)^{n} \frac{1}{\left|B_{\frac{R}{2}}-\Omega_{k_{t}, \frac{R}{2}}\right|} 2|\nabla w|_{A ; D_{t}} \cdot|1|_{\widetilde{A} ; D_{t}} \\
\leq & C_{1} 2^{t} \psi^{-1} R^{n}\left(2^{-1} \omega_{n}\left(2^{-1} R\right)^{n}\right)^{-1} \mathfrak{A}^{-1}\left(\int_{D_{t}} A(x,|\nabla w|) \mathrm{d} x\right) \cdot \widehat{\widehat{\mathfrak{A}}}^{-1}\left(\int_{D_{t}} \widetilde{A}(x, 1) \mathrm{d} x\right) \\
\leq & c 2^{t} \psi^{-1} \mathfrak{A}^{-1}\left(\int_{D_{t}} A(x,|\nabla w|) \mathrm{d} x\right) \cdot \widehat{\widetilde{\mathfrak{A}}}^{-1}\left(\left|D_{t}\right|\right) .
\end{aligned}
$$




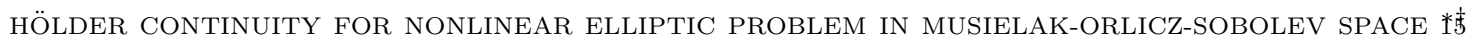

From Lemma 3.1. Remark 3.1, $\mathfrak{A}^{-1}, \mathfrak{A} \in \Delta_{\mathbb{R}^{+}}$, (3.40) and (3.39) we can conclude

$$
\begin{aligned}
\left|\Omega_{k_{s-1}, \frac{R}{2}}\right|^{1-\frac{1}{n}} & \leq c 2^{t} \psi^{-1} \mathfrak{A}^{-1}\left(c \gamma \widehat{\mathfrak{A}}\left(R^{-1} 2^{-t} \psi\right) R^{n}\right) \cdot \widehat{\widehat{\mathfrak{A}}}^{-1}\left(\left|D_{t}\right|\right) \\
& \leq c\left(R 2^{t} \psi^{-1}\right) \mathfrak{A}^{-1}\left(\widehat{\mathfrak{A}}\left(R^{-1} 2^{-t} \psi\right)\right) R^{-1} \mathfrak{A}^{-1}\left(c \gamma R^{n}\right) \cdot \widehat{\widetilde{\mathfrak{A}}}^{-1}\left(\left|D_{t}\right|\right) \\
& \leq c_{1} R^{-1} \mathfrak{A}^{-1}\left(c \gamma R^{n}\right) \cdot \widehat{\widehat{\mathfrak{A}}}^{-1}\left(\left|D_{t}\right|\right) .
\end{aligned}
$$

From (3.41) and $\widehat{\widetilde{\mathfrak{A}}} \in \Delta_{R^{+}}$we have

$$
\widehat{\widetilde{\mathfrak{A}}}\left(\left|\Omega_{k_{s-1}, \frac{R}{2}}\right|^{1-\frac{1}{n}}\right) \leq c_{0} \widehat{\widetilde{\mathfrak{A}}}\left(c_{1} R^{-1} \mathfrak{A}^{-1}\left(c \gamma R^{n}\right)\right) \cdot\left|D_{t}\right| .
$$

Summing (3.42) with respect to $t$ from 0 to $s-2$ and noting that

$$
\sum_{t=0}^{s-2}\left|D_{t}\right| \leq\left|B_{\frac{R}{2}}\right|=\omega_{n}\left(\frac{R}{2}\right)^{n}
$$

we obtain

$$
\widehat{\widetilde{\mathfrak{A}}}\left(\left|\Omega_{k_{s-1}, \frac{R}{2}}\right|^{1-\frac{1}{n}}\right) \leq \frac{c_{0} 2^{-n} \omega_{n}}{s-1} \widehat{\widetilde{\mathfrak{A}}}\left(c_{1} R^{-1} \mathfrak{A}^{-1}\left(c \gamma R^{n}\right)\right) R^{n} .
$$

By Lemma 3.1, $\mathfrak{A}^{-1}, \mathfrak{A} \in \Delta_{\mathbb{R}^{+}}$, 3.43) and Remark 3.1, we can get

$$
\begin{aligned}
\left|\Omega_{k_{s-1}, \frac{R}{2}}\right|^{1-\frac{1}{n}} & \leq M_{1} \widehat{\widetilde{\mathfrak{A}}}^{-1}\left(\frac{c_{0} 2^{-n} \omega_{n}}{s-1}\right) c_{1} R^{-1} \mathfrak{A}^{-1}\left(c \gamma R^{n}\right) \widehat{\widetilde{\mathfrak{A}}}^{-1}\left(R^{n}\right) \\
& =M_{1} \widehat{\widetilde{\mathfrak{A}}}^{-1}\left(\frac{c_{0} 2^{-n} \omega_{n}}{s-1}\right) c_{1} R^{-1} \mathfrak{A}^{-1}\left(c \gamma R^{n}\right) R^{n} \mathfrak{A}^{-1}\left(R^{-n}\right) \\
& \leq M_{2} \widehat{\widetilde{\mathfrak{A}}}^{-1}\left(\frac{c_{0} 2^{-n} \omega_{n}}{s-1}\right) c_{1} R^{n-1} \mathfrak{A}^{-1}(c \gamma) \mathfrak{A}^{-1}\left(R^{n}\right) \mathfrak{A}^{-1}\left(R^{-n}\right) \\
& \leq M_{3} \widehat{\widetilde{\mathfrak{A}}}^{-1}\left(\frac{c_{0} 2^{-n} \omega_{n}}{s-1}\right) R^{n-1},
\end{aligned}
$$

and therefore

$$
\left|\Omega_{k_{s-1}, \frac{R}{2}}\right| \leq\left(M_{3} \widehat{\widetilde{\mathfrak{A}}}^{-1}\left(\frac{c_{0} 2^{-n} \omega_{n}}{s-1}\right)\right)^{\frac{n}{n-1}} R^{n} .
$$

where $M_{3}=M_{3}(\gamma, n, A)$ and $c_{0}=c_{0}(A)$ are constants.

Now we choose a natural number $s$ such that $s-1>c_{0} 2^{-n} \omega_{n}$ and

$$
\left(M_{3} \widehat{\widetilde{\mathfrak{A}}}^{-1}\left(\frac{c_{0} 2^{-n} \omega_{n}}{s-1}\right)\right)^{\frac{n}{n-1}}<\theta
$$

note that $s=s\left(\theta, n, M_{3}, c_{0}, \widehat{\widetilde{\mathfrak{A}}}^{-1}\right)=s(n, \gamma, A, \theta)$. For such $s$, if (3.36) does not hold, then from (3.44) we get

$$
\left|\Omega_{k_{s-1}, \frac{R}{2}}\right| \leq \theta R^{n},
$$

which means $\left|\Omega_{k^{0}, \frac{R}{2}}\right| \leq \theta R^{n}$, i.e. (3.37) holds since

$$
k^{0}=\max _{B_{R}} w(x)-2^{-s+1} \psi=k_{s-1} .
$$


Lemma 3.8. There is a number $s=s(n, A, \gamma)>2$ such that

$$
\psi \leq 2^{s} \max \left\{\max _{B_{R}} w(x)-\max _{B_{\frac{R}{4}}} w(x), R \widehat{\mathfrak{A}}^{-1}\left(\frac{\gamma_{1}}{\gamma}\right)\right\}
$$

for any $R \in\left(0, R_{0}\right]$.

Proof. Let $R \in\left(0, R_{0}\right]$ and let $\theta=\theta(n, A, \gamma)$ be the constant as in Lemma 3.6 Applying Lemma 3.7 to this $\theta$ we can find a constant $s=s(\theta, n, A, \gamma)=s(n, A, \gamma)$ such that at least one of (3.36) and (3.37) holds.

If (3.36) holds, then (3.45) is obviously true.

Now assume that (3.36) does not hold. Then Lemma 3.7 (3.37) holds, i.e.

$$
\left|\Omega_{k^{0}, \frac{R}{2}}\right| \leq \theta R^{n},
$$

where $k^{0}=\max _{B_{R}} w(x)-2^{-s+1} \psi$. Set $H=2^{-s+1} \psi$. Since (3.36) does not hold, we get

By Lemma 3.6

$$
H>2^{-s+1} \cdot 2^{s} R \widehat{\mathfrak{A}}^{-1}\left(\frac{\gamma_{1}}{\gamma}\right)>R \widehat{\mathfrak{A}}^{-1}\left(\frac{\gamma_{1}}{\gamma}\right) .
$$

i.e.

$$
\max _{B_{R / 4}} w(x) \leq k^{0}+\frac{H}{2}=\max _{B_{R}} w(x)-2^{-s} \psi
$$

$$
\psi \leq 2^{s}\left(\max _{B_{R}} w(x)-\max _{R / 4} w(x)\right) .
$$

This shows that equation (3.45) holds.

Lemma 3.8 is proved.

Lemma 3.9. For any $R \in\left(0, R_{0}\right]$ at least one of the following two inequalities holds

$$
\begin{gathered}
\operatorname{osc}\left\{u, B_{R}\right\} \leq \tau 2^{s} \widehat{\mathfrak{A}}^{-1}\left(\frac{\gamma_{1}}{\gamma}\right) R, \\
\operatorname{osc}\left\{u, B_{R / 4}\right\} \leq\left(1-\tau^{-1} 2^{-s}\right) \operatorname{osc}\left\{u, B_{R}\right\},
\end{gathered}
$$

where $\tau=\max \left\{2, \frac{2}{\delta}\right\}$, and $s=s(n, A, \gamma)$ is the constant as in Lemma 3.8.

Proof. By Lemma 3.8, equation (3.45) holds. Then at least one of the following two inequalities holds

$$
\begin{gathered}
\psi \leq 2^{s} R \widehat{\mathfrak{A}}^{-1}\left(\frac{\gamma_{1}}{\gamma}\right), \\
\psi \leq 2^{s}\left(\max _{B_{R}} w(x)-\max _{R / 4} w(x)\right) .
\end{gathered}
$$

When equation (3.48) holds we have

$$
\operatorname{osc}\left\{u ; B_{R}\right\}=\tau \psi \leq \tau 2^{s} R \widehat{\mathfrak{A}}^{-1}\left(\frac{\gamma_{1}}{\gamma}\right)
$$

i.e. (3.46) holds. 
When (3.49) holds we have

$$
\begin{aligned}
\operatorname{osc}\left\{u ; B_{R}\right\} & =\tau \psi \\
& \leq \tau 2^{s}\left(\max _{B_{R}} w(x)-\max _{R / 4} w(x)\right) \\
& \leq \tau 2^{s}\left(\max _{B_{R}} w(x)-\max _{R / 4} w(x)-\min _{B_{R}} w(x)+\min _{R / 4} w(x)\right) \\
& \leq \tau 2^{s}\left[\operatorname{osc}\left\{w ; B_{R}\right\}-\operatorname{osc}\left\{w ; B_{R / 4}\right\}\right] . \\
& =\tau 2^{s}\left[\operatorname{osc}\left\{u ; B_{R}\right\}-\operatorname{osc}\left\{u ; B_{R / 4}\right\}\right],
\end{aligned}
$$

which implies that (3.47) holds.

Lemma 3.9 is proved.

The proof of Theorem 3.1. By Lemma 3.4 and it follows from Lemma 3.9 that $u \in C^{0, \alpha}\left(B_{R_{0}}\left(x_{0}\right)\right)$ where

$$
\alpha=\min \left\{1,-\log _{4}\left(1-\tau^{-1} 2^{-s}\right)\right\}=-\log _{4}\left(1-\tau^{-1} 2^{-s}\right)=\alpha(n, A, \gamma, \delta) .
$$

By the arbitrarily of $x_{0} \in \Omega$ we have $u \in C^{0, \alpha}(\Omega)$. The proof of Theorem 3.1 is completed.

\section{Application to minimizers}

Consider the integral functionals as follows

$$
E(v)=E(v, \Omega)=\int_{\Omega} f(x, v(x), \nabla v(x)) \mathrm{d} x
$$

where $v \in W^{1, A}(\Omega)$ and $f(x, s, z)$ is a Carathéodory function on $\Omega \times \mathbb{R} \times \mathbb{R}^{n}$ satisfying

$$
A\left(x, \sum_{i=1}^{n}\left|z_{i}\right|\right)-B(x, s)-b \leq f(x, s, z) \leq a\left(A\left(x, \sum_{i=1}^{n}\left|z_{i}\right|\right)+B(x, s)+b\right)
$$

with $a$ and $b$ being non-negative constants, $A \in N(\Omega) \cap \mathscr{A}$ satisfying $\left(A_{1}\right)$ and $\left(C_{1}\right)$ (see In Section 3), $N(\Omega) \ni B \preccurlyeq A_{*}$ satisfying the following $\left(B_{1}\right)-\left(B_{2}\right)$ :

$\left(B_{1}\right)$ There exists a strictly increasing differentiable function $\mathfrak{B}:[0,+\infty) \rightarrow$ $[0,+\infty)$ satisfying

$$
B(x, \alpha t) \geq \mathfrak{B}(\alpha) B(x, t), \forall \alpha \geq 0, t \in \mathbb{R}, x \in \Omega ;
$$

$\left(B_{2}\right)$ There exists a constant $T_{B, \Omega}>0$ such that $B\left(x, T_{B, \Omega}\right) \geq 1$ for any $x \in \bar{\Omega}$.

Definition 4.1. A function $u \in W_{l o c}^{1, A}(\Omega)$ is said to be a local minimizer of $E$ if

$$
E(u ; \operatorname{supp} \varphi) \leq E(u+\varphi ; \operatorname{supp} \varphi) \text { for any } \varphi \in W_{0}^{1, A}(\Omega) \text { with supp } \varphi \subset \subset \Omega \text {. }
$$

Theorem 4.1. Let $f$ satisfy the growth condition (4.2). If $u \in W^{1, A}(\Omega)$ is a local minimizer for the functional (4.1), then

(i) $u \in L_{l o c}^{\infty}(\Omega)$;

(ii) $u \in C^{0, \alpha}(\Omega)$ in which the constant $\alpha \in(0,1)$ depends only on $n, A$ and $a$. 
The assertion (i) of Theorem 4.1 has been obtained by Theorem 4.1 of [?].

The assertion (ii) of Theorem 4.1 follows from Theorem 3.1 and the following Lemma 4.1.

Lemma 4.1. Under the assumptions of Theorem 4.1, for any open set $\Omega_{1}$ in $\Omega$ with $\bar{\Omega}_{1} \subset \Omega$, there exist positive constants $M, \gamma$ and $\gamma_{1}$ such that $u \in \mathscr{B}\left(\Omega_{1}, M, \gamma, \gamma_{1}, 1\right)$ in which the constant $\gamma=\gamma(a, A)$ is independent of $\Omega_{1}$.

Proof. Let $\Omega_{1}$ be an open set in $\Omega$ with $\Omega_{1} \subset \Omega$. By assertion $(i)$ of Theorem 4.1 there is a constant $M>0$ such that

$$
\max _{\bar{\Omega}_{1}} u(x) \leq M
$$

From (4.2) it follow that there is a positive constant $c=c\left(a, b, B_{+}, M\right)=$ $c(a, b, A, M)$ such that

$$
A\left(x, \sum_{i=1}^{n}\left|z_{i}\right|\right)-c \leq f(x, u, z) \leq a A\left(x, \sum_{i=1}^{n}\left|z_{i}\right|\right)+c, \forall x \in \bar{\Omega}_{1}, \quad|u| \leq 4 M, \quad z \in R^{n},
$$

where $B_{+}=\max _{\bar{\Omega}_{1}} B(x, M) \leq \max _{\bar{\Omega}_{1}} A_{*}(x, M)+C \leq C_{0}$.

Let $B_{s} \subset \Omega_{1}, 0<t<s, k \geq-2 M, w(x)=\max \{u(x)-k, 0\}$. Choose $\eta \in C^{\infty}$ with supp $\eta \subset B_{s}, 0 \leq \eta \leq 1, \eta \equiv 1$ on $B_{t},|D \eta| \leq 2(s-t)^{-1}$. Set $v=u-\eta w$. Then for $x \in \bar{\Omega}_{1}$

$$
|v(x)| \leq|u(x)|+|w(x)| \leq M+3 M=4 M .
$$

By the minimality of $u$ and (4.5) we get

$$
\begin{aligned}
& \int_{\Omega_{k, s}} A(x,|\nabla u|) \mathrm{d} x-c\left|\Omega_{k, s}\right| \\
\leq & a \int_{\Omega_{k, s}} A(x,|\nabla v|) \mathrm{d} x+c\left|\Omega_{k, s}\right| \\
\leq & \frac{a}{2} \widehat{\mathfrak{A}}(2)\left[\int_{\Omega_{k, s}} A(x,|\nabla u|(1-\eta)) \mathrm{d} x+\int_{\Omega_{k, s}} A(x,|\nabla \eta|(u-k)) \mathrm{d} x\right]+c\left|\Omega_{k, s}\right| \\
\leq & \frac{a}{2} \widehat{\mathfrak{A}}(2) \int_{\Omega_{k, s} \backslash \Omega_{k, t}} A(x,|\nabla u|) \mathrm{d} x+\frac{a}{2}(\widehat{\mathfrak{A}}(2))^{2} \int_{\Omega_{k, s}} A\left(x, \frac{u-k}{s-t}\right) \mathrm{d} x+c\left|\Omega_{k, s}\right| .
\end{aligned}
$$

Therefore

$$
\begin{aligned}
& \int_{\Omega_{k, t}} A(x,|\nabla u|) \mathrm{d} x \\
\leq & \int_{\Omega_{k, s}} A(x,|\nabla u|) \mathrm{d} x \\
\leq & \frac{a}{2} \widehat{\mathfrak{A}}(2) \int_{\Omega_{k, s} \backslash \Omega_{k, t}} A(x,|\nabla u|) \mathrm{d} x+\frac{a}{2}(\widehat{\mathfrak{A}}(2))^{2} \int_{\Omega_{k, s}} A\left(x, \frac{u-k}{s-t}\right) \mathrm{d} x+2 c\left|\Omega_{k, s}\right| .
\end{aligned}
$$


Adding $\frac{a}{2} \widehat{\mathfrak{A}}(2) \int_{\Omega_{k, t}} A(x,|\nabla u|) \mathrm{d} x$ to both sides of (4.7) we get

$$
\begin{aligned}
& \int_{\Omega_{k, t}} A(x,|\nabla u|) \mathrm{d} x \\
\leq & \theta \int_{\Omega_{k, s}} A(x,|\nabla u|) \mathrm{d} x+c_{1} \int_{\Omega_{k, s}} A\left(x, \frac{u-k}{s-t}\right) \mathrm{d} x+c_{2}\left|\Omega_{k, s}\right|,
\end{aligned}
$$

where $\theta=\frac{a \widehat{\mathfrak{A}}(2)}{2+a \widehat{\mathfrak{A}}(2)}<1, c_{1}=c_{1}(a, A)$ and $c_{2}=c_{2}(c, a, A)=c_{2}(a, b, A, M)$ are positive constants. Using the similar method that was used in the proof of Lemma 4.1 of [?], from (4.8) we can deduce that

$$
\int_{\Omega_{k, \sigma R}} A(x,|\nabla u|) \mathrm{d} x \leq \gamma \int_{\Omega_{k, R}} A\left(x, \frac{u-k}{(1-\sigma) R}\right) \mathrm{d} x+\gamma_{1}\left|\Omega_{k, s}\right|
$$

for $B_{R} \subset \Omega_{1}, \sigma \in(0,1)$ and $k \geq-2 M$, where $\gamma=\gamma(a, A)$ and $\gamma_{1}=\gamma_{1}(a, b, A, M)$ are positive constants.

Set $\delta=1$. Then for every $B_{\rho} \subset \Omega_{1}$

$$
\max _{B_{\rho}} u(x)-M \geq-2 M,
$$

and consequently (4.9) holds for $k \geq \max _{B_{R}} u(x)-\delta M$ with $\delta=1$.

It is easy to see that (4.9) holds when $u$ is replaced by $-u$. So by Definition 3.2 $u \in \mathscr{B}\left(\Omega_{1}, M, \gamma, \gamma_{1}, 1\right)$. Lemma 4.1 is proved.

The proof of Theorem 4.1. In the case $A$ satisfies $\left(A_{1}\right)$ and $\left(C_{1}\right)$, by Lemma 4.1 and Theorem 3.1, $u \in C^{0, \alpha}\left(\bar{\Omega}_{1}\right)$ for every $\bar{\Omega}_{1} \subset \Omega$ and the constant $\alpha \in(0,1)$ is independent of $\Omega_{1}$. Hence $u \in C^{0, \alpha}(\Omega)$. The proof of Theorem 4.1 is completed.

\section{Application to FUlly nOnlinear elliptic EQUATIONS}

In this section, we consider the local Hölder continuity of weak solutions of a kind of fully nonlinear elliptic equation. Since we only consider the local properties of the weak solutions, without loss of generality, we suppose that $\Omega$ is a bounded smooth domain in $\mathbb{R}^{n}$.

Consider the second order fully nonlinear elliptic equation as follows

$$
\operatorname{div} L(x, u, \nabla u)+F(x, u, \nabla u)=0, \forall x \in \Omega,
$$

where $L: \Omega \times \mathbb{R} \times \mathbb{R}^{n} \rightarrow \mathbb{R}^{n}, F: \Omega \times \mathbb{R} \times \mathbb{R}^{n} \rightarrow \mathbb{R}^{1}$, and $u: \Omega \rightarrow \mathbb{R}$.

Suppose equation (5.1) satisfies the following growth conditions:

$$
\begin{gathered}
L(x, u, z) z \geq a_{0} A(x,|z|)-b B(x, u)-c, \\
|L(x, u, z)| \leq a_{1} \widetilde{A}^{-1} A(x,|z|)+b \widetilde{A}^{-1} B(x, u)+c, \\
|F(x, u, z)| \leq a_{2} \widetilde{B}^{-1} A(x,|z|)+b \widetilde{B}^{-1} B(x, u)+c,
\end{gathered}
$$

where $a_{0}, a_{1}, a_{2}, b, c$ are positive constants, $A \in N(\Omega) \cap \mathscr{A}$ satisfies $\left(A_{1}\right)$ and $\left(C_{1}\right)$, $N(\Omega) \ni B \preccurlyeq A_{*}$ satisfies $\left(B_{1}^{+}\right)-\left(B_{2}^{+}\right)$. 
$\left(B_{1}^{+}\right)$There exists a strictly increasing differentiable function $\mathfrak{B}:[0,+\infty) \rightarrow$ $[0,+\infty)$ satisfying

$$
\alpha \mathfrak{B}^{\prime}(\alpha)>\mathfrak{B}(\alpha)
$$

such that

$$
B(x, \alpha t) \geq \mathfrak{B}(\alpha) B(x, t), \forall \alpha \geq 0, t \in \mathbb{R}, x \in \Omega
$$

$\left(B_{2}^{+}\right)$There exists a constant $T_{B, \Omega}>0$ such that $B\left(x, T_{B, \Omega}\right) \geq 1$ for any $x \in \bar{\Omega}$.

Definition 5.1. $u \in W^{1, A}(\Omega)$ is said to be a weak solution of (5.1) if

$$
\int_{\Omega} L(x, u, \nabla u) \nabla v \mathrm{~d} x-\int_{\Omega} F(x, u, \nabla u) v \mathrm{~d} x=0
$$

for any $v \in W_{0}^{1, A}(\Omega)$.

The local bounded regularity of weak solutions of (5.1) satisfying (5.2)-(5.4) has been obtained by Theorem 5.1 of [?]. Now we discuss the Hölder continuity of weak solutions of (5.1).

Theorem 5.1. Let the growth conditions (5.2)-(5.4) hold. If $u \in W^{1, A}(\Omega)$ is a weak solution of (5.1) and $\max _{\Omega}|u(x)| \leq M$, then $u \in \mathscr{B}\left(\Omega, M, \gamma, \gamma_{1}, \delta\right)$ in which $\gamma=\gamma\left(a_{0}, a_{1}, A\right), \gamma_{1}=\gamma_{1}\left(a_{0}, a_{2}, b, c, A, M\right), \delta=\min \left\{\frac{a_{0}}{4 a_{2} M}, 2\right\}$.

Proof. Let $u$ be a weak solution of (5.1). For arbitrary balls $\bar{B}_{s} \subset B_{t} \subset \Omega$ and pick a function $\xi \in C^{\infty}(\Omega)$ such that

$$
0 \leq \xi \leq 1, \quad \operatorname{supp} \xi \subset B_{t}, \quad \xi \equiv 1 \text { on } B_{s}, \quad|D \xi| \leq \frac{2}{t-s} .
$$

Let

$$
\delta=\min \left\{\frac{a_{0}}{4 a_{2} M}, 2\right\},
$$

and $v=\mathfrak{A}(\xi) \max \{u-k, 0\} \in W_{0}^{1, A}(\Omega)$, where

$$
k \geq \max _{B_{t}} u(x)-\delta M .
$$

By (5.6) we obtain

$$
\begin{array}{r}
\int_{\Omega_{k, t}} \mathfrak{A}(\xi) L(x, u, \nabla u) \cdot \nabla u \mathrm{~d} x+\int_{\Omega_{k, t}}(u-k) L(x, u, \nabla u) \cdot \nabla \mathfrak{A}(\xi) \mathrm{d} x \\
-\int_{\Omega_{k, t}} \mathfrak{A}(\xi)(u-k) F(x, u, \nabla u) \mathrm{d} x=0 .
\end{array}
$$


HÖLDER CONTINUITY FOR NONLINEAR ELLIPTIC PROBLEM IN MUSIELAK-ORLICZ-SOBOLEV SPACE $2 \hat{1}$ From (5.2)-(5.4) and (5.10), it follows that

$$
\begin{aligned}
& a_{0} \int_{\Omega_{k, t}} A(x,|\nabla u|) \mathfrak{A}(\xi) \mathrm{d} x \leq b \int_{\Omega_{k, t}} B(x,|u|) \mathfrak{A}(\xi) \mathrm{d} x+c \int_{\Omega_{k, t}} \mathfrak{A}(\xi) \mathrm{d} x \\
& \quad+a_{1} \int_{\Omega_{k, t}} \widetilde{A}^{-1} A(x,|\nabla u|)|\nabla \mathfrak{A}(\xi)|(u-k) \mathrm{d} x \\
& \quad+b \int_{\Omega_{k, t}} \widetilde{A}^{-1} B(x,|u|)|\nabla \mathfrak{A}(\xi)|(u-k) \mathrm{d} x+c \int_{\Omega_{k, t}}|\nabla \mathfrak{A}(\xi)|(u-k) \mathrm{d} x \\
& \quad+a_{2} \int_{\Omega_{k, t}} \widetilde{B}^{-1} A(x,|\nabla u|) \mathfrak{A}(\xi)(u-k) \mathrm{d} x \\
& \quad+b \int_{\Omega_{k, t}} \widetilde{B}^{-1} B(x,|u|) \mathfrak{A}(\xi)(u-k) \mathrm{d} x+c \int_{\Omega_{k, t}} \mathfrak{A}(\xi)(u-k) \mathrm{d} x .
\end{aligned}
$$

We will estimate each term of the right-hand side of (5.11). By $B \preccurlyeq A_{*}$ and Remark 2.1 we obtain

$$
\begin{aligned}
b \int_{\Omega_{k, t}} B(x,|u|) \mathfrak{A}(\xi) \mathrm{d} x & \leq b \int_{\Omega_{k, t}} B(x, M) \mathfrak{A}(1) \mathrm{d} x \\
& \leq b \int_{\Omega_{k, t}} A_{*}(x, M) \mathfrak{A}(1) \mathrm{d} x+b \int_{\Omega_{k, t}} C \mathfrak{A}(1) \mathrm{d} x \\
& \leq C_{0}\left|\Omega_{k, t}\right|
\end{aligned}
$$

where $C_{0}=C_{0}(b, A, M)$, and

$$
c \int_{\Omega_{k, t}} \mathfrak{A}(\xi) \mathrm{d} x \leq c \int_{\Omega_{k, t}} \mathfrak{A}(1) \mathrm{d} x=c \mathfrak{A}(1)\left|\Omega_{k, t}\right| .
$$

By the Young inequality, and taking $\epsilon>0$ such that

$$
a_{1} \widetilde{\mathfrak{A}}(n \epsilon)=\frac{a_{0}}{4}
$$

we deduce from the assumption $n \mathfrak{A}(\alpha)>\alpha \mathfrak{A}^{\prime}(\alpha)>\mathfrak{A}(\alpha)$ and Lemma 3.1 (iii) that $(5.14)$

$$
\begin{aligned}
& a_{1} \int_{\Omega_{k, t}} \widetilde{A}^{-1} A(x,|\nabla u|)|\nabla \mathfrak{A}(\xi)|(u-k) \mathrm{d} x \\
= & a_{1} \int_{\Omega_{k, t}} \widetilde{A}^{-1} A(x,|\nabla u|)|\nabla \xi| \mathfrak{A}^{\prime}(\xi)(u-k) \mathrm{d} x \\
\leq & a_{1} \int_{\Omega_{k, t}} \widetilde{A}\left(x, \epsilon \widetilde{A}^{-1} A(x,|\nabla u|) \mathfrak{A}^{\prime}(\xi)\right) \mathrm{d} x+a_{1} \int_{\Omega_{k, t}} A\left(x, \epsilon^{-1}|\nabla \xi|(u-k)\right) \mathrm{d} x \\
\leq & a_{1} \int_{\Omega_{k, t}} \widetilde{A}\left(x, \epsilon \widetilde{A}^{-1} A(x,|\nabla u|) \frac{n \mathfrak{A}(\xi)}{\xi}\right) \mathrm{d} x+a_{1} \int_{\Omega_{k, t}} A\left(x, \epsilon^{-1}|\nabla \xi|(u-k)\right) \mathrm{d} x \\
\leq & a_{1} \widetilde{\mathfrak{A}}(n \epsilon) \int_{\Omega_{k, t}} A(x,|\nabla u|) \mathfrak{A}(\xi) \mathrm{d} x+a_{1} \widehat{\mathfrak{A}}\left(\frac{2}{\epsilon}\right) \int_{\Omega_{k, t}} A\left(x, \frac{u-k}{t-s}\right) \mathrm{d} x \\
= & \frac{a_{0}}{4} \int_{\Omega_{k, t}} A(x,|\nabla u|) \mathfrak{A}(\xi) \mathrm{d} x+a_{1} \widehat{\mathfrak{A}}\left(\frac{2}{\epsilon}\right) \int_{\Omega_{k, t}} A\left(x, \frac{u-k}{t-s}\right) \mathrm{d} x .
\end{aligned}
$$


WANG, LIU, AND ZHAO

By the Young inequality, (5.8) and (5.9) we obtain that

$$
\begin{aligned}
& a_{2} \int_{\Omega_{k, t}} \widetilde{B}^{-1} A(x,|\nabla u|) \mathfrak{A}(\xi)(u-k) \mathrm{d} x \\
\leq & a_{2} \int_{\Omega_{k, t}} A(x,|\nabla u|) \mathfrak{A}(\xi)(u-k) \mathrm{d} x+a_{2} \int_{\Omega_{k, t}} B(x, 1) \mathfrak{A}(\xi)(u-k) \mathrm{d} x \\
\leq & a_{2} \int_{\Omega_{k, t}} A(x,|\nabla u|) \mathfrak{A}(\xi)\left(\max _{B_{t}} u(x)-k\right) \mathrm{d} x+a_{2} \int_{\Omega_{k, t}} B(x, 1) \mathfrak{A}(1)\left(\max _{B_{t}} u(x)-k\right) \mathrm{d} x \\
\leq & a_{2} \delta M \int_{\Omega_{k, t}} A(x,|\nabla u|) \mathfrak{A}(\xi) \mathrm{d} x+a_{2} \delta M \mathfrak{A}(1)\left(\int_{\Omega_{k, t}} A_{*}(x, 1) \mathrm{d} x+\int_{\Omega_{k, t}} C \mathrm{~d} x\right) \\
\leq & \frac{a_{0}}{4} \int_{\Omega_{k, t}} A(x,|\nabla u|) \mathfrak{A}(\xi) \mathrm{d} x+C_{1}\left|\Omega_{k, t}\right|,
\end{aligned}
$$

where $C_{1}=C_{1}\left(a_{0}, A\right)$. Using Young's inequality and taking $\varepsilon_{2} \in(0,1)$ such that

$$
b \widehat{\mathfrak{A}}\left(2 \varepsilon_{2}\right)=1,
$$

we deduce from the assumption $n \mathfrak{A}(\alpha)>\alpha \mathfrak{A}^{\prime}(\alpha)>\mathfrak{A}(\alpha)$ and Lemma 3.1 (iii) that

$$
\begin{aligned}
& b \int_{\Omega_{k, t}} \widetilde{A}^{-1} B(x,|u|)|\nabla \mathfrak{A}(\xi)|(u-k) \mathrm{d} x \\
= & b \int_{\Omega_{k, t}} \widetilde{A}^{-1} B(x,|u|)|\nabla \xi| \mathfrak{A}^{\prime}(\xi)(u-k) \mathrm{d} x \\
\leq & b \int_{\Omega_{k, t}} \widetilde{A}\left(x, \varepsilon_{2}^{-1} \widetilde{A}^{-1} B(x,|u|) \mathfrak{A}^{\prime}(\xi)\right) \mathrm{d} x+b \int_{\Omega_{k, t}} A\left(x, \varepsilon_{2}|\nabla \xi|(u-k)\right) \mathrm{d} x \\
\leq & b \int_{\Omega_{k, t}} \widetilde{A}\left(x, \varepsilon_{2}^{-1} \widetilde{A}^{-1} B(x,|u|) \frac{n \mathfrak{A}(\xi)}{\xi}\right) \mathrm{d} x+b \widehat{\mathfrak{A}}\left(2 \varepsilon_{2}\right) \int_{\Omega_{k, t}} A\left(x, \frac{u-k}{t-s}\right) \mathrm{d} x \\
\leq & b \widetilde{\mathfrak{A}}\left(\varepsilon_{2}^{-1} n\right) \int_{\Omega_{k, t}} B(x, M) \mathfrak{A}(\xi) \mathrm{d} x+b \widehat{\mathfrak{A}}\left(2 \varepsilon_{2}\right) \int_{\Omega_{k, t}} A\left(x, \frac{u-k}{t-s}\right) \mathrm{d} x \\
\leq & C_{2}\left|\Omega_{k, t}\right|+\int_{\Omega_{k, t}} A\left(x, \frac{u-k}{t-s}\right) \mathrm{d} x,
\end{aligned}
$$

where $C_{2}=C_{2}(b, A, n, M)$. Using Young's inequality we get

$$
\begin{aligned}
& c \int_{\Omega_{k, t}}|\nabla \mathfrak{A}(\xi)|(u-k) \mathrm{d} x \\
= & c \int_{\Omega_{k, t}}|\nabla \xi| \mathfrak{A}^{\prime}(\xi)(u-k) \mathrm{d} x \\
\leq & 2 \int_{\Omega_{k, t}} A\left(x, \frac{u-k}{t-s}\right) \mathrm{d} x+2 \int_{\Omega_{k, t}} \widetilde{A}\left(x, c \mathfrak{A}^{\prime}(\xi)\right) \mathrm{d} x \\
\leq & 2 \int_{\Omega_{k, t}} A\left(x, \frac{u-k}{t-s}\right) \mathrm{d} x+\int_{\Omega_{k, t}} \widetilde{A}\left(x, c \frac{n \mathfrak{A}(\xi)}{\xi}\right) \mathrm{d} x \\
\leq & 2 \int_{\Omega_{k, t}} A\left(x, \frac{u-k}{t-s}\right) \mathrm{d} x+\int_{\Omega_{k, t}} \widetilde{A}(x, c n) \mathfrak{A}(\xi) \mathrm{d} x \\
\leq & 2 \int_{\Omega_{k, t}} A\left(x, \frac{u-k}{t-s}\right) \mathrm{d} x+C_{3}\left|\Omega_{k, t}\right|,
\end{aligned}
$$


where $C_{3}=C_{3}(c, n, A)$. Similarly, we have

$$
\begin{aligned}
& b \int_{\Omega_{k, t}} \widetilde{B}^{-1} B(x,|u|) \mathfrak{A}(\xi)(u-k) \mathrm{d} x \\
\leq & b \int_{\Omega_{k, t}} B(x,|u|) \mathrm{d} x+b \int_{\Omega_{k, t}} B(x, \mathfrak{A}(\xi)(u-k)) \mathrm{d} x \\
\leq & b \int_{\Omega_{k, t}} B(x, M) \mathrm{d} x+b \int_{\Omega_{k, t}} B(x, \delta M \mathfrak{A}(1)) \mathrm{d} x \\
\leq & b \int_{\Omega_{k, t}} B(x, M) \mathrm{d} x+b \int_{\Omega_{k, t}} B\left(x, \frac{a_{0}}{4 a_{2}} \mathfrak{A}(1)\right) \mathrm{d} x \\
\leq & C_{4}\left|\Omega_{k, t}\right|
\end{aligned}
$$

where $C_{4}=C_{4}\left(b, A, M, a_{0}, a_{2}\right)$.

$$
c \int_{\Omega_{k, t}} \mathfrak{A}(\xi)(u-k) \mathrm{d} x \leq c \int_{\Omega_{k, t}} \mathfrak{A}(1) \delta M \mathrm{~d} x \leq \frac{c \mathfrak{A}(1) a_{0}}{4 a_{2}}\left|\Omega_{k, t}\right| .
$$

From (5.11)-(5.19) we conclude that

$$
\int_{\Omega_{k, t}} A(x,|\nabla u|) \mathfrak{A}(\xi) \mathrm{d} x \leq \gamma \int_{\Omega_{k, t}} A\left(x, \frac{u-k}{t-s}\right) \mathrm{d} x+\gamma_{1}\left|\Omega_{k, t}\right| .
$$

Therefore

$$
\mathfrak{A}(1) \int_{\Omega_{k, s}} A(x,|\nabla u|) \mathrm{d} x \leq \gamma \int_{\Omega_{k, t}} A\left(x, \frac{u-k}{t-s}\right) \mathrm{d} x+\gamma_{1}\left|\Omega_{k, t}\right|,
$$

for $\bar{B}_{s} \subset B_{t} \subset \Omega$ and $k$ satisfying (5.9), where $\gamma=\gamma\left(a_{0}, a_{1}, n, A\right)$ and $\gamma_{1}=$ $\gamma_{1}\left(a_{0}, a_{2}, b, c, A, n, M\right)$ are positive constants. (5.20) shows that $u \in \mathscr{B}\left(\Omega, M, \gamma, \gamma_{1}, \delta\right)$. Theorem 5.1 is proved.

From Theorems 5.1 and 3.1 we obtain

Theorem 5.2. Suppose that the assumptions of Theorems 5.1 hold. If $u \in W^{1, A}(\Omega)$ is a weak solution of (5.1) and $\max _{\Omega}|u(x)| \leq M$, then $u \in C^{0, \alpha}(\Omega)$ in which $\alpha=\alpha\left(a_{0}, a_{1}, \delta, A, n\right)=\alpha\left(a_{0}, a_{1}, a_{2}, M, A, n\right) \in(0,1)$.

\section{REFERENCES}

[1] E. Acerbi and N. Fusco. Regularity for minimizers of non-quadratic functionals: The case $1<p<2$. J. Math. Anal. Appl., 140:115-135, 1989.

[2] E. Acerbi and N. Fusco. Partial regularity under anisotropic $(p, q)$ growth conditions. J. Differential Equations, 107:46-67, 1994.

[3] E. Acerbi and N. Fusco. A transmission problem in the calculus of variations. Calc. Var., 2:1-16, 1994.

[4] T. Adamowicz and O. Toivanen. Hölder continuity of quasiminimizers with nonstandard growth. Nonlinear Analysis, 125:433-456, 2015.

[5] R. Adams. Sobolev Spaces. Acad. Press, New York, 1975.

[6] P. Baroni, M. Colombo, and G. Mingione. Non-autonomous functionals: borderline cases and related function classes. St. Petersburg Math. J., 27:347-379, 2016.

[7] M. Colombo and G. Mingione. Bounded minimizers of double phase variational integrals. Arch. Ration. Mech. Anal., 218:219-273, 2015.

[8] M. Colombo and G. Mingione. Regularity for double phase variational problems. Arch. Ration. Mech. Anal., 215:443-496, 2015.

[9] A. Coscia and G. Mingione. Hölder continuity of the gradient of $p(x)$-harmonic mappings. $C$. R. Acad. Sci. Paris Ser. I Math., 328:363-368, 1999.

[10] E. De Giorgi. Sulla differenziabilità e l'analiticità delle estremali degli integrali multipli regolari. Mem. Accad. Sci. Torino, 3(3):25-43, 1957. 
[11] L. Diening, P. Hästö, and S. Roudenko. Function spaces of variable smoothness and integrability. J. Func. Anal., 256:1731-1768, 2009.

[12] L. Diening, B. Stroffolini, and A. Verde. Everywhere regularity of functionals with $\varphi$-growth. Manuscripta Math., 129:449-481, 2009.

[13] L. Diening, B. Stroffolini, and A. Verde. Lipschitz regularity for some asymptotically convex problems. ESAIM: Control, Optimisation and Calculus of Variations, 17:178-189, 2011.

[14] T. K. Donaldson and N. S. Trudinger. Orlicz-sobolev spaces and imbedding theorems. J. Func. Anal., 8:52-75, 1971.

[15] Acerbi E. and G. Mingione. Regularity results for a class of functionals with non-standard growth. Arch. Ration. Mech. Anal., 156:121-140, 2001.

[16] X. Fan and D. Zhao. A class of de giorgi type and Hölder continuous. Nonlinear Analysis, 36:295-318, 1999.

[17] X. L. Fan. Differential equations of divergence form in Musielak-Sobolev spaces and subsupersolution method. J. Math. Anal. Appl., 386:593-604, 2012

[18] X. L. Fan. An imbedding theorem for Musielak-Sobolev spaces. Nonlinear Analysis, 75:19591971, 2012.

[19] M. Giaquinta. Multiple Integrals in the Calculus of Variations and Nonlinear Elliptic Systems. Princeton University Press, Princeton, 1983.

[20] M. Giaquinta. Growth conditions and regularity, a counterexample. Manuscr. Math., 59:245$248,1987$.

[21] M. Giaquinta and E. Giusti. On the regularity of the minima of variational integrals. Acta Math., 148:31-46, 1982.

[22] P. Harjulehto, P. Hästö, and O. Toivanen. Hölder regularity of quasiminimizers under generalized growth conditions. Calc. Var., 56(22), 2017.

[23] O.A. Ladyzhenskaya and N.N. Ural'tseva. Linear and Quasilinear Elliptic Equations. Nauka, Moscow, 1973.

[24] G. M. Lieberman. The natural generalization of the natural conditions of Ladyzhenskaya and Uraltseva for elliptic equations. Comm. PDE, 16:311-361, 1991.

[25] G. M. Lieberman. Gradient estimates for a class of elliptic systems. Ann. Mat. Pura Appl. (IV), 164:103-120, 1993.

[26] G. M. Lieberman. Gradient estimates for a new class of degenerate elliptic and parabolic equations. Ann. Scuola Norm. Sup. Pisa Cl. Sci. (IV), 21:497-522, 1994.

[27] G. M. Lieberman. Gradient estimates for anisotropic elliptic equations. Adv. Diff. Equ., 10:767-782, 1994.

[28] D. Liu and P. Zhao. Solutions for a quasilinear elliptic equation in Musielak-Sobolev spaces. Nonlinear Analysis: Real World Applications, 26:315-329, 2015.

[29] J. J. Manfredi. Regularity of the gradient for a class of nonlinear possibly degenerate elliptic equations. Ph.D. Thesis. University of Washington, St. Louis, 1986.

[30] J. J. Manfredi. Regularity for minima of functionals with p-growth. J. Differ. Equ., 76:203$212,1988$.

[31] P. Marcellini. Regularity of minimizers of integrals of the caculus of variations with nonstandard growth conditions. Arch. Rat. Mech. Anal., 105:267-284, 1989.

[32] P. Marcellini. Regularity and existence of solutions of elliptic equations with $p, q$-growth conditions. J. Differential Equations, 90:1-30, 1991.

[33] P. Marcellini. Regularity for elliptic equations with general growth conditions. J. Differ. Equ., 105:296-333, 1993.

[34] G. Mingone. Regularity of minima: an invitation to the dark side of the calculus of variations. Appl. Math., 51:355-425, 2006.

[35] C.B. Morrey. Multiple Integrals in the Calculus of Variations. Springer, Berlin, 1968.

[36] J. Musielak. Orlicz Spaces and Modular Spaces, in: Lecture Notes in Math., volume 1034. Springer-Verlag, Berlin, 1983.

[37] T. Schmidt. Regularity of relaxed minimizers of quasiconvex variational integrals with $(p, q)-$ growth. Arch. Ration. Mech. Anal., 193:311-337, 2008.

[38] T. Schmidt. Regularity of minimizers of $W^{1, p}$-quasiconvex variational integrals with $(p, q)$ growth. Calc. Var. PDE, 32:1-24, 2009.

(Beibei Wang) School of Mathematics and Statistics, Lanzhou University, Lanzhou 730000, P. R. CHINA

E-mail address: wangbb15@lzu.edu.cn

(Duchao Liu) School of Mathematics and Statistics, Lanzhou University, Lanzhou

730000, P. R. CHINA

E-mail address: liuduchao@gmail.com, Tel.: +8613893289235, fax: +8609318912481

(Peihao Zhao) School of Mathematics and Statistics, Lanzhou University, Lanzhou 730000, P. R. CHINA

E-mail address: zhaoph@lzu.edu.cn 